\title{
Vulnérabilité des terres des écosystèmes du domaine soudanien au Bénin de 1995 à 2015
}

\section{Fiacre Codjo Ahononga ${ }^{1}$ Gérard Nounagnon GouWAKINNOU ${ }^{1}$ Samadori Sorotori Honoré BIAOU ${ }^{1}$ Séverin BIAOU ${ }^{1}$}

${ }^{1}$ Laboratoire d'écologie, de botanique et de biologie végétale

Université de Parakou

03 BP 125, Parakou

République du Bénin

\section{Auteur correspondant /}

Corresponding author:

Fiacre Codjo AHONONGA -

fiacreahononga@gmail.com

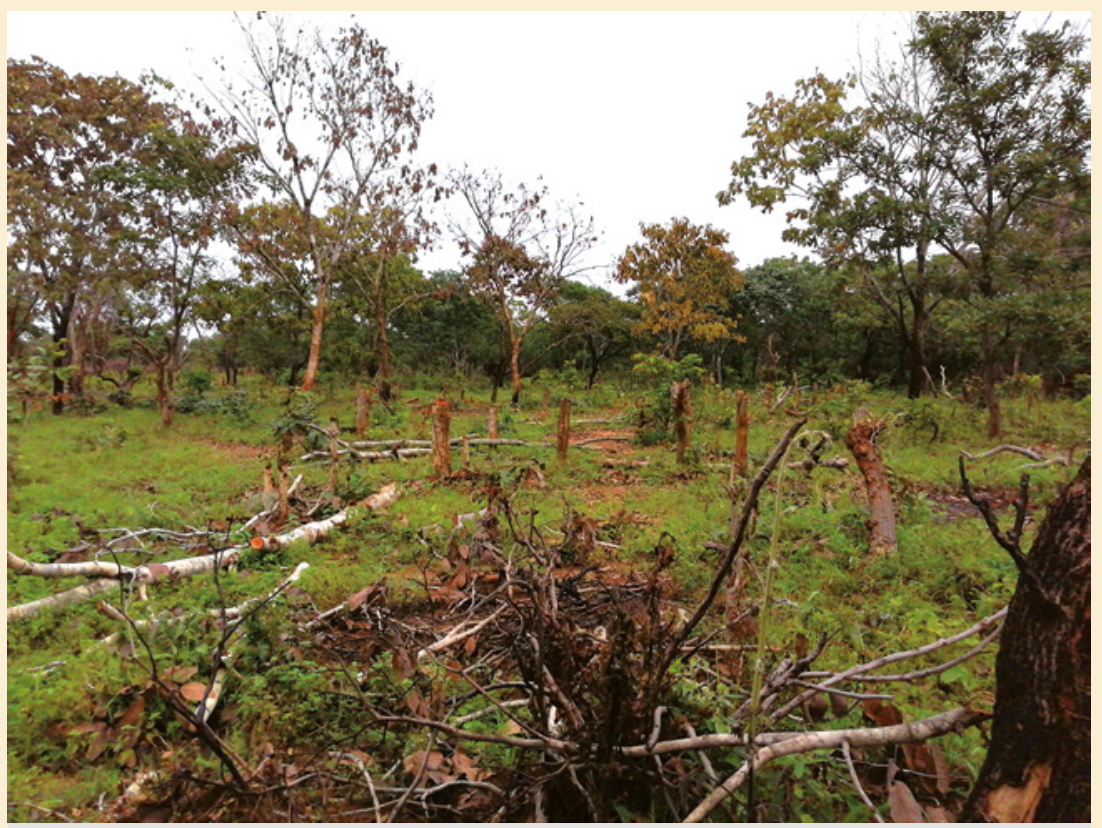

Photo 1.

Défrichement dans un une aire protégée.

Photo F. Ahononga.

Doi : 10.19182/bft2020.346.a36295 - Droit d'auteur (c) 2020, Bois et Forêts des Tropiques - (c) Cirad - Date de soumission : 5 décembre 2019 ; date d'acceptation : 14 août 2020 ; date de publication : 25 décembre 2020.

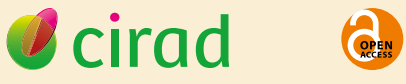

Licence Creative Commons :

Attribution - 4.0 International.

Attribution-4.0 International (CC BY 4.0)
Citer l'article / To cite the article

Ahononga F. C., Gouwakinnou G. N., Biaou S. S. H., Biaou S., 2020. Vulnérabilité des terres des écosystèmes du domaine soudanien au Bénin de 1995 à 2015. Bois et Forêts des Tropiques, 346 : 35-50. Doi : https://doi.org/10.19182/bft2020.346.a36295 


\section{RÉSUMÉ}

\section{Vulnérabilité des terres des écosystèmes du domaine soudanien au Bénin de 1995 à 2015}

Le Nord du Bénin abrite $92,5 \%$ de la couverture forestière nationale, principalement constituée des réserves de faune et de forêts classées, destinées à la conservation de la biodiversité. Ces zones du domaine classé, pourtant dotées pour la plupart de plans d'aménagement et d'un système de surveillance qui devraient limiter la dégradation du milieu, sont soumises à un fort taux de régression de la couverture forestière, dû aux pressions anthropiques. Cette étude vise à analyser la vulnérabilité des formations forestières dans les domaines classé et non classé de trois communes du Nord-Bénin : Matéri, Toucountouna et Ségbana. Une interprétation visuelle des images SPOT de 1995, 2005 et 2015, avec le logiciel Quantum GIS, a été effectuée pour faire ressortir les changements et évaluer la vulnérabilité des formations forestières. Les résultats révèlent une régression des formations forestières naturelles au profit de celles anthropiques. Les champs et jachères ont vu leur superficie multipliée par près de cinq entre 2005 et 2015. Au cours de cette même période, la dégradation a été plus forte dans le domaine classé que dans le non classé, alors qu'à l'inverse la déforestation a surtout affecté le domaine non classé. Les formations naturelles ont été vulnérables à la perte de superficie au profit des champs et jachères et des habitations. Les taux de vulnérabilité des différentes formations sont fonction de la période et du niveau de protection. Ces situations constituent une menace pour la biodiversité et nécessitent la mise en place d'une politique de planification de l'occupation des terres dans la périphérie du domaine classé. Cela garantira leur fonction et réduira les effets des facteurs de déforestation et de dégradation sur les réserves forestières.

Mots-clés : dégradation, déforestation, domaine non classé, domaine classé, vulnérabilité, Bénin.

\section{ABSTRACT}

\section{Vulnerability of Sudanian ecosystem lands in Benin from 1995 to 2015}

Northern Benin has $92.5 \%$ of the country's entire forest cover, mainly made up of wildlife and forest reserves for biodiversity conservation. Although most of these protected forests have management plans and surveillance systems in place that should limit environmental damage, the forest cover is shrinking rapidly due to human pressures. This study aimed to analyse the vulnerability of forest stands in protected and unprotected zones in three municipalities in northern Benin: Matéri, Toucountouna and Ségbana. SPOT images from 1995 , 2005 and 2015 were interpreted visually with Quantum GIS software to highlight changes and assess the vulnerability of the forest stands. The results show that natural forest stands are losing ground to human land uses. The area of fields and fallow lands increased almost fivefold from 2005 to 2015. During the same period, land degradation was greater in the protected zones than in the non-protected zones. Conversely, deforestation mainly affected the non-protected zones. Natural tree stands were vulnerable to losing surface area to fields, fallow land and homes. The rates of vulnerability of the different stands varied with the period considered and according to the level of protection. These situations are a threat to biodiversity and demand the introduction of a land use planning policy for areas around the protected zones, in order to safeguard their functions and reduce the effects of deforestation and land degradation on the forest reserves.

Keywords: degradation, deforestation, non-protected area, protected area, vulnerability, Benin.

\section{RESUMEN}

\section{Vulnerabilidad de las tierras en los ecosistemas del dominio sudanés de Benín de 1995 a 2015}

El norte de Benín alberga el $92,5 \%$ de la cobertura boscosa nacional, constituida principalmente por reservas de fauna $y$ de bosques protegidos, destinados a la conservación de la biodiversidad. Estas zonas del dominio protegido, en su mayor parte con planes de ordenación y un sistema de vigilancia que deberían limitar la degradación del medio, sufren una fuerte tasa de regresión de la cobertura boscosa, debida a las presiones antrópicas. El objetivo de este estudio es analizar la vulnerabilidad de las formaciones forestales en los dominios protegidos y no protegidos de tres municipios del norte de Benín: Matéri, Toucountouna y Ségbana. Se realizó una interpretación visual de las imágenes SPOT de 1995, 2005 y 2015 con el software Quantum GIS, para destacar los cambios y evaluar la vulnerabilidad de las formaciones forestales. Los resultados revelan una regresión de formaciones forestales naturales a favor de las antrópicas. Los campos y barbechos multiplicaron su superficie casi por cinco entre 2005 y 2015. En el curso de este mismo período, la degradación fue mayor en el dominio protegido que en el no protegido, mientras que, a la inversa, la deforestación afectó especialmente al dominio no protegido. Las formaciones naturales fueron vulnerables ante la pérdida de superficie en beneficio de los campos, de los barbechos y de las viviendas. Las tasas de vulnerabilidad de las diferentes formaciones son función del período y del nivel de protección. Estas situaciones constituyen una amenaza para la biodiversidad y requieren la aplicación de una política de planificación de la ocupación de las tierras alrededor de la periferia del dominio protegido. Ello garantizará su función y reducirá los efectos de los factores de deforestación y de degradación en las reservas forestales.

Palabras clave: degradación, deforestación, dominio no protegido, dominio protegido, vulnerabilidad, Benín. 


\section{Introduction}

Les écosystèmes forestiers constituent le pilier essentiel du maintien de l'équilibre global de la planète. Ils soutiennent la biodiversité terrestre à plus de 80 \% (Déclaration de New York sur les forêts, 2014 ; UNFCC, 2014) et contribuent au maintien des services écosystémiques (IPBES, 2019). Les forêts participent également au développement socio-économique à travers leur contribution à la sécurité alimentaire, la santé et l'amélioration des relations sociales (Kaboré, 2015 ; Baudron et al., 2019 ; Rasmussen et al., 2020). Les Nations unies reconnaissent la forêt à juste titre comme un élément indispensable dans l'atteinte des Objectifs de développement durable (ODD). Malgré cette importance reconnue, les écosystèmes forestiers sont soumis à une surexploitation qui provoque leur dégradation graduelle. En effet, les études récentes font état d'un recul du couvert forestier terrestre, passant de $31,6 \%$ en 1990 à 30,6 \% en 2015 (FAO, 2018). La perte annuelle nette des forêts tropicales a été estimée à sept millions d'hectares entre 2000 et 2010 (FAO, 2016). Ce fort taux de déforestation enregistré dans les forêts tropicales menace non seulement la biodiversité (Reidsma et al., 2006 ; Liang et Liu, 2017 ; Liu et al., 2019) mais aussi perturbe la régulation du carbone libéré dans l'atmosphère (Polasky et al., 2011 ; Pareta et Pareta, 2011 ; Gouwakinnou et al., 2018 ; Brandt et al., 2018). Le changement d'affectation des terres contribue fortement à la modification de l'environnement. La forme la plus répandue de changement d'utilisation des terres est l'expansion agricole car plus d'un tiers de la superficie terrestre est utilisé pour les cultures et l'élevage (IPBES, 2019). La situation est encore plus alarmante dans les pays du Sud où les actions de la population sont plus orientées vers l'exploitation de la ressource que vers la conservation. Ainsi, le couvert forestier de l'Afrique subsaharienne est passé de 30,6 \% à 27,1\% entre 1990 et 2015 (FAO, 2018). Cette régression du couvert végétal due aux activités humaines pour la satisfaction des besoins socio-économiques est la cause du changement de la structure spatiale des paysages. Plusieurs études aux niveaux régional et local confirment ces transformations qui induisent la fragmentation du paysage forestier (Oloukoi, 2013 ; Avakoudjo et al., 2015). Une telle fragmentation fragilise la structure des forêts et crée un dysfonctionnement des écosystèmes forestiers (Benbrahim et al., 2004). Ces problèmes écologiques sont majoritairement les conséquences de l'exploitation agropastorale, l'agriculture itinérante sur brûlis et la croissance démographique (Mama et al., 2013).

Le Bénin, l'un des pays de l'Afrique de l'Ouest dont le développement est basé sur la l'agriculture, n'échappe pas à cette situation. À l'expansion agricole et la croissance démographique (taux d'accroissement de la population de $3,5 \%$; INSAE, 2016) s'ajoute également la baisse de la pluviométrie (Tidjani et Akponikpe, 2012).

Le Nord-Bénin est caractérisé par un climat soudanien. Cette portion du pays abrite $92,5 \%$ de la couverture forestière nationale (Hountondji, 2008). Elle est constituée des réserves de faune et de forêts classées, destinées à la conservation de la biodiversité. Pourtant, cette zone septentrionale présente un fort taux de régression de la couverture végétale, due aux pressions anthropiques. Ces dernières aboutissent à une destruction importante du paysage forestier se traduisant par une régression des formations naturelles au profit des champs et jachères avec une désorganisation de la configuration du paysage (Mama et al., 2013 ; Avakoudjo et al., 2015). Cette régression semble s'observer aussi bien dans le domaine classé que non classé (Ousseni et al., 2016 ; Biaou et al., 2019) ; alors que les forêts classées sont dotées pour la plupart de plans d'aménagement et d'un système de surveillance qui devrait limiter la dégradation du milieu. Il est rappelé que le domaine classé correspond à des espaces appartenant à l'État, définitivement affectés à la forêt et ayant fait l'objet de délimitation et de classement ou de reconnaissance officielle. À l'inverse, le domaine non classé correspond au domaine forestier national susceptible d'être affecté à des utilisations autres que forestières (agriculture, élevage, habitation) ; les forêts communautaires et des particuliers font également partie du domaine non classé.

Ainsi, l'efficacité des plans d'aménagement et des systèmes de surveillance, comme outils de préservation de la biodiversité, peut-elle être légitimement interrogée. La limite des connaissances sur le processus d'affectation des terres dans le domaine classé comparé au domaine non classé pour la conservation de la biodiversité handicape les mécanismes de gestion durable du domaine classé. Cette connaissance permettrait de mieux planifier ce domaine afin d'y garantir la conservation de la biodiversité.

Dans ce contexte, l'objectif de cette étude est d'analyser par télédétection les changements d'affectation des terres et la vulnérabilité des unités d'occupation du sol dans le domaine soudanien du Bénin suivant le gradient de protection de l'écosystème forestier, afin de concevoir une stratégie de gestion des réserves forestières qu'elles abritent.

\section{Matériel et méthodes}

\section{Zone d’étude}

L'étude a été conduite sur trois communes du NordBénin, dans les départements de l'Alibori et de l'Atacora compris entre $10^{\circ} 30^{\prime} 00^{\prime \prime}$ et $11^{\circ} 45^{\prime} 00^{\prime \prime}$ de latitude Nord et entre $2^{\circ} 3^{\prime} 00^{\prime \prime}$ et $3^{\circ} 16^{\prime} 40^{\prime \prime}$ de longitude Est. Les communes de la zone d'étude (Ségbana, 447292 ha ; Toucountouna, 110895 ha ; Matéri, 171887 ha) sont situées respectivement dans les districts phytogéographiques du Borgou, au nord de la chaîne de l'Atacora et du Mékrou-Pendjari (Adomou, 2005). (figure 1). Cette zone présente un régime pluviométrique unimodal avec un gradient septentrional 
allant de 1200 mm à 900 mm (Adomou, 2005). L'humidité de l'air varie de $18 \%$, pendant la saison à laquelle souffle l'harmattan (vent soufflant du nord-est de décembre à février), à $99 \%$ en août pendant la saison des pluies. La température moyenne mensuelle se situe entre $24^{\circ} \mathrm{C}$ et $31{ }^{\circ} \mathrm{C}$ (Adomou, 2005). Cette zone est caractérisée par la prédominance des exploitations agricoles et sujette aux aléas climatiques. L'agriculture est la principale activité des populations. Elle est de type extensif avec une dominance des cultures de l'igname et du coton. Dans le département de l'Alibori, le poids démographique était de $8,7 \%$ de la population du Bénin en 2013 (contre 7,7 \% au recensement de 2002). Quant au département de l'Atacora, il est moins peuplé, avec 7,7\% de la population du Bénin en 2013 contre 8,1 \% en 2002 (INSAE, 2016).

L'ensemble de la zone étudiée comprend une partie de la réserve cynégétique de la Pendjari et les réserves forestières classées de Sota et des Trois Rivières (figure 1).

Les trois domaines classés de la zone d'étude sont constitués de mosaïques de forêts claires (avec éventuellement des forêts denses sèches) parsemées de savanes arborées et arbustives et traversées par des galeries forestières et des plantations forestières (White, 1983). Différents types de sols y sont présents : sols minéraux peu évolués et peu fertiles, ferrugineux sur socle cristallin de fertilité variable ; hydromorphes ; lithosols; cuirasses ferrallitiques.

\section{Données satellitaires et auxiliaires}

Les images satellitaires SPOT utilisées (1995, 2005 et 2015) couvrant les trois communes étudiées sont celles acquises par le Centre national d'études spatiales (CNES) et Airbus et livrées au projet Observation spatiale des forêts d'Afrique centrale et de l'Ouest $\left(\mathrm{OSFACO}^{1}\right)$. La disponibilité et la qualité ont été les critères de choix de ces images. Des images étant indisponibles et de mauvaise qualité aux dates à considérer pour l'étude, le choix s'est reporté sur celles disponibles et de qualité exploitable les plus proches dans l'année précédente ou la suivante. Les images sont issues des capteurs HRV1 (scènes : 065-327, 065-328, 065-329, 064-327, 060-328, 060-329, 059328, 060-329 du 19/12/1994; 20 m de résolution spatiale), HRV1 (scènes : $20 \mathrm{~m}$ de résolution spatiale), HRVIR2 (scènes : 067-327, 068-327, 067-328, 068-328, 068-328 du 25/01/2005 au 03/03/2005; 20 m de résolution spatiale), HRG1 (scènes : 063-329, 063-328, 064-329 du $19 / 12 / 2005 ; 10 \mathrm{~m}$ de résolution spatiale) et MS (scènes : 1948980101, 1948950101, 1948936101, 1948935101, 1948979101, 1948981101, 1948940101, 1948948101 du 02/12/2014 au 01/03/2015, 6 m de résolution spatiale). Les images considérées sont toutes enregistrées en saison sèche. Aussi, diverses données auxiliaires dont

${ }^{1}$ www.osfaco.org

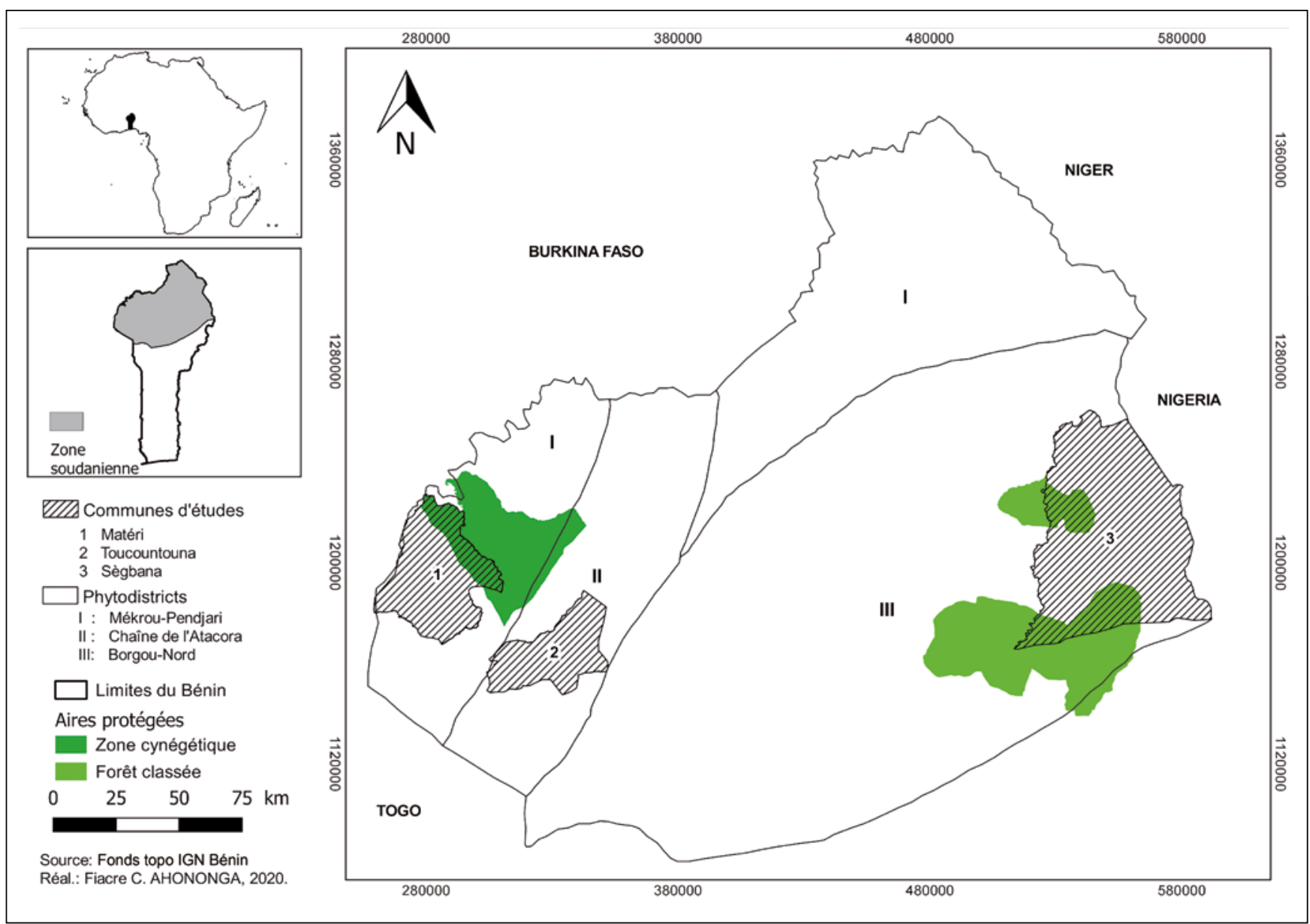

Figure 1.

Localisation de la zone d'étude. 
notamment les feuilles de découpage de cartes topographiques du Bénin (échelle 1/50 000), le réseau routier, les cours d'eau et les limites administratives ont été utilisées. À cela s'ajoutent les données issues de la mission de reconnaissance terrain qui s'est déroulée du 27 août au $1^{\text {er }}$ septembre 2018 et les données de validation qui ont été recueillies entre le 17 et le 23 décembre 2018. Ces points de contrôle et de reconnaissance ont été collectés à l'aide d'un GPS Garmin 78. La mission de reconnaissance a permis de collecter des données au niveau de 244 points échantillons et 325 points complémentaires sur le terrain, soit au total 569 points. Six cent huit points ont été tirés aléatoirement dans les différentes classes identifiées pour en faire la vérification sur le terrain.

\section{Méthodes de traitement des images satellitaires et de validation de l'interprétation}

\section{Prétraitement d'images}

Toutes les images ont subi une correction radiométrique, atmosphérique et de géoréférencement dans le référentiel UTM-31 WGS-84 Nord par le fournisseur avant acquisition. Ces opérations sont réalisées afin d'augmenter la lisibilité des images, de faciliter leur interprétation (Biaou et al., 2019) et de rendre superposables les images issues des différentes dates. Pour rendre homogène la taille des pixels à $6 \mathrm{~m}$ de résolution, les pixels des images SPOT de 1995 et 2005 ont été également ré-échantillonnées par le fournisseur avant acquisition. Les extractions et des mosaïques d'images ont été appliquées.

\section{Interprétation visuelle}

Dans cette étude, l'interprétation visuelle des images à l'écran a été adoptée pour analyser les images SPOT de $6 \mathrm{~m}$ de résolution. Cette technique a été choisie pour sa performance dans l'analyse des images basées sur la connaissance du terrain et la signature spectrale (ton, forme, texture et structure). Bien que moins rapide et exigeant une activité de terrain intense, plusieurs études y ont eu recours pour affiner la technique de classification supervisée (Mama et al., 2013 ; Mamane et al., 2018 ; Sangne et al., 2019 ; Bah et al., 2019). Cette méthode qui tire profit de la signature spectrale est la plus proche de la méthode traditionnelle de la photo-interprétation. Elle est reconnue robuste pour l'identification et la caractérisation des unités spatiales sur une image (Bah et al., 2019 ; Biaou et al., 2019), le cerveau humain étant un bon interprète d'images (Bah et al., 2019). La photo-interprétation permet de limiter les confusions spectrales de certaines classes lors de la classification supervisée. En outre, plusieurs auteurs (Mama et Oloukoi, 2003 ; Barima et al., 2009 ; Mama et al., 2013 ; Mamane et al., 2018 ; Bah et al., 2019 ; Barima et al., 2019) ont utilisé cette méthode pour affiner les images traitées par la méthode de classification supervisée. Certaines classes confondues à travers la signature spectrale lors de la classification supervisée peuvent être séparées par l'interprétation visuelle (Bah et al., 2019).

L'interprétation a été effectuée sous Quantum GIS
(Quantum GIS, 2013). Une composition colorée en fausses couleurs associant les canaux aux trois couleurs rouge, verte et bleue de l'écran a été appliquée respectivement pour l'infrarouge (canal 3), le rouge (canal 2) et le vert (canal 1) afin de faciliter le regroupement des pixels dans les différentes classes d'occupation du sol (Sikuzani et al., 2019). L'interprétation a permis d'opérer une stratification des zones homogènes et de leur affecter une classe d'occupation du sol (Hammi et al., 2007 ; Biaou et al., 2019). La délimitation des entités de différentes classes s'est basée sur la signature spectrale des unités d'occupation du sol (la couleur, le ton, la structure, la texture, la forme et la localisation). De plus, les images d'archives de Google Earth pour les années 1995 et 2005 et les points de reconnaissance sur le terrain pour l'image de 2015 ont renforcé l'interprétation visuelle. La démarche adoptée pour la reconnaissance du terrain est basée sur le ralliement des points échantillonnés à partir de leurs coordonnées géographiques à l'aide du GPS Garmin 78. L'interprétation des images a débuté avec celles de 2015 à partir desquelles celles de 2005 ont été interprétées. Cela consistait à copier les attributs de 2015 dans les champs d'attribut de 2005 et à procéder ensuite à des modifications par l'interprétation des images de 2005. De la même manière, les images de 1995 ont été interprétées à partir de celles de 2005.

\section{Évaluation de la précision}

Elle consiste à déterminer de façon quantitative l'efficacité avec laquelle les pixels sont regroupés dans de bonnes classes d'occupation du sol de la zone d'étude (Batar et al., 2017). Pour cette étude, les données de référence des années 1995 et 2005 ont été collectées à partir des images d'archives de Google Earth correspondant à ces dates. En ce qui concerne les données de référence de l'année 2015, les 608 points d'intérêt dans les différentes classes identifiées ont été aléatoirement relevés en 2018 au cours de la saison sèche (du 17 au 23 décembre 2018). Pour valider l'interprétation visuelle, la matrice de confusion a été établie. L'extension "QChainage " a permis d'échantillonner de façon aléatoire les points de contrôle. L'extension "Point Sampling Tool » du logiciel Quantum GIS a été utilisée pour extraire automatiquement à partir des fichiers de forme l'occupation du sol à chacun des points échantillonnés. Les coordonnées de chacun de ces points ont été extraites grâce à la calculatrice de champs du logiciel pour obtenir un tableau de points composant l'échantillon. Ce tableau a servi de base pour le contrôle sur le terrain. Cela a permis d'établir le tableau de confusion 2015. L'application logicielle "QFIELD », sous le système d'exploitation Android, a été utilisée pour les vérifications terrain. Elle intègre l'occupation du sol, les points échantillons et le formulaire de validation sur le terrain. Pour les dates historiques, les images d'archive Google Earth et Bing Aerial ont été utilisées afin de confondre les données théoriques issues de l'interprétation visuelle des images SPOT. Les matrices de confusion ont été un outil pratique pour calculer les erreurs de l'interprétation par les méthodes conventionnelles, notamment les erreurs de commission et d'omission. 
La précision globale de chaque classe ainsi que le coefficient de Kappa (K) ont été calculés. Les équations 1 et 2 présentent respectivement la méthode de calcul de la précision globale et du coefficient de Kappa :

$a=\frac{1}{N} \sum_{i=1}^{N c} x i i$ (équation 1)

$K=\frac{a-b}{1-b} \quad$ avec $b=\frac{1}{N^{2}} \sum_{i=1}^{N c}\left(x_{+i} \cdot x_{i+}\right)$

(équation 2)

avec Nc: nombre total de classes ; $N$ : nombre total de points d'observation ; xii : nombre de points d'observation dans la colonne i et ligne i correspondant à la diagonale de la matrice $; \mathrm{x}_{+\mathrm{i}}$ : total de points d'observation dans la colonne $i$ (total à droite dans la matrice) ; $x_{i+}$ : total de points d'observation dans la ligne i (total en bas de la matrice) ; a : précision globale.

L'intervalle de confiance (Ic) à $95 \%$ est calculé comme suit :

$I c=\sqrt{a} \frac{1-a}{N} \star 1,96$

(équation 3)

avec Ic : intervalle de confiance à $95 \%$; a : précision globale ; $\mathrm{N}$ : nombre total d'observations.

\section{Exportation vers un système d'information géographique}

Chacune des images a été importée dans le logiciel ArcGIS 10.3.1. Ces dernières ont été vectorisées afin de visualiser les interprétations puis de calculer des statistiques au niveau des types de classes pour chacune des trois dates. Les cartes d'occupation du sol des trois dates des zones à accès restreints appelées domaine classé (les forêts classées de Sota et des Trois Rivières, et la zone cynégétique de la Pendjari) ont été extraites afin de comparer leur dynamique aux zones d'accès libre à la communauté (domaine non classé).

\section{Analyse de la dynamique de l'occupation du sol}

La dynamique spatiale est basée sur l'appréciation de l'évolution du couvert forestier et la comparaison de la superficie des différentes unités d'occupation du sol de chaque zone. Pour l'analyse statistique de la dynamique d'occupation du sol, le taux d'évolution de chaque classe (unité paysagère) a été calculé d'une date à une autre. Il est obtenu à partir de la matrice de transition (Coulibaly et al., 2016) :

$\operatorname{Tv}(\%)=\left[\frac{S 2}{S 1}-1\right] \times 100 \quad$ (équation 4)

avec Tv : taux d'évolution de la classe ; S1 : superficie de l'unité d'occupation du sol à l'année 1 ; S2 : superficie de l'unité d'occupation du sol à l'année 2.

Ainsi, si $\mathrm{S} 2-\mathrm{S} 1<0$, cela correspond à une régression du couvert végétal de l'année 1 à 2 . Si S2 - S1 > 0, cela correspond à une augmentation du couvert végétal de l'année 1 à 2 . Si S2 $-\mathrm{S} 1=0$, cela correspond à une stabilité du couvert végétal de l'année 1 à 2 .

\section{Analyse de la vulnérabilité, de la déforestation et de la dégradation des écosystèmes}

La matrice de transition entre deux dates a permis d'évaluer les pertes et les gains de surface au sein de chaque unité d'occupation du sol. Ces superficies ont été utilisées pour évaluer l'intensité des changements. La vulnérabilité au changement de chaque classe d'occupation du sol a été calculée à l'aide respectivement du ratio gain/ stabilité $(\mathrm{Gs}=\mathrm{g} / \mathrm{s})$, du ratio perte/stabilité $(P s=p / s)$ et $d u$ ratio changement net/stabilité (Ns $=\mathrm{Gs}-\mathrm{SPs}$ ). Les termes $g$, $s$ et $p$ représentent respectivement les gains, les zones de stabilité et les pertes de superficie de chaque occupation du sol. Quand certaines classes d'occupation du sol présentent des valeurs de Gs, Ps plus élevées que d'autres, alors elles ont une plus forte probabilité au changement qu'à la stabilité dans leurs conditions. Si la valeur de Ns est négative, la classe d'occupation du sol aura alors une forte probabilité de perdre des superficies au profit des autres classes qui en gagnent (Biaou et al., 2019).

Pour l'analyse de la déforestation et la dégradation forestière au sein des écosystèmes, des regroupements des transitions de classes d'occupation du sol ont été inspirés de Wasseige et al. (2009). Ils ont permis de les définir et de les interpréter en catégorie de changement. La diminution du couvert végétal des formations forestières naturelles (FFN) est considérée comme une dégradation. La conversion des FFN en formations non forestières (FNF) représente la déforestation. La conversion des FFN et FNF en plantation représente une reconstitution du couvert forestier. Ainsi, le taux global de déforestation ( $\operatorname{Tg}($ Def) $)$ est calculé par la formule de Wasseige et al. (2009) utilisée par Tchatchou et al. (2015). Le taux annuel de déforestation et de dégradation a été obtenu respectivement en divisant le taux de déforestation et de dégradation par le nombre d'années d'étude :

$T g(D e f)=\frac{D e f}{S 1} \times 100 \quad$ (équation 5)

où Def $(b ; n)$ est la déforestation brute (b) ou nette (n) et $\mathrm{S} 1$ représente la superficie des formations forestières naturelles (FFN) de l'année t1.

Le taux global de dégradation $\operatorname{Tg}(\mathrm{Dg})$ est calculé par la formule suivante :

$\operatorname{Tg}(D g)=\frac{D g}{S 1} \times 100$

(équation 6)

où $\mathrm{Dg}(\mathrm{b} ; \mathrm{n})$ est la dégradation brute $(\mathrm{b})$ ou nette $(\mathrm{n})$ et $\mathrm{S} 1$ représente la superficie des formations forestières naturelles (FFN) de l'année t1.

\section{Analyse des données}

Le taux d'évolution, de vulnérabilité des différents types de formations a été comparé entre les deux types de domaines (classé et non classé) en fonction de la période. À cet effet, des graphiques de type "boîte à moustaches " ont été réalisés. 


\section{Résultats}

\section{Cartographie et validation de l'interprétation visuelle}

La discrimination des unités homogènes à travers l'interprétation visuelle a été significative, car l'indice de Kappa est de 0,89, 0,92 et 0,95 respectivement pour les années 1995, 2005 et 2015. La précision globale de l'interprétation des images de 1995, 2005 et 2015 est respectivement de $91,05 \%, 93,68 \%$ et $96 \%$ avec un intervalle de confiance de 1,58 (tableaux I, II et III). Bien qu'on constate certaines confusions entre les classes d'occupation du sol, les résultats

Figure 2.

Cartes de l'occupation du sol des écosystèmes de Ségbana en 1995 , 2005 et 2015.

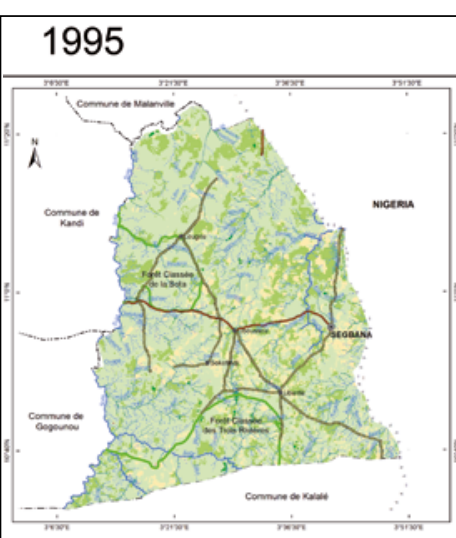

2005

- ${ }^{e}$ trimestre - décembre 2020 - p. 35-50 VULNÉRABILITÉ DES TERRES / LE POINT SUR.

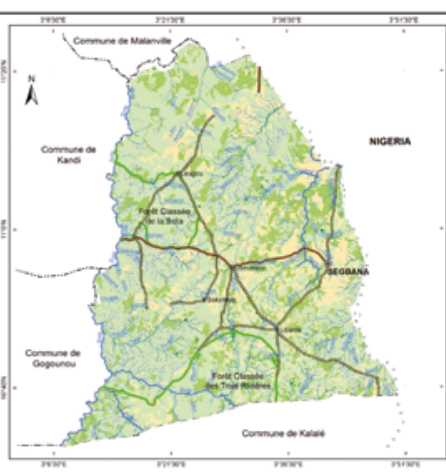

2015

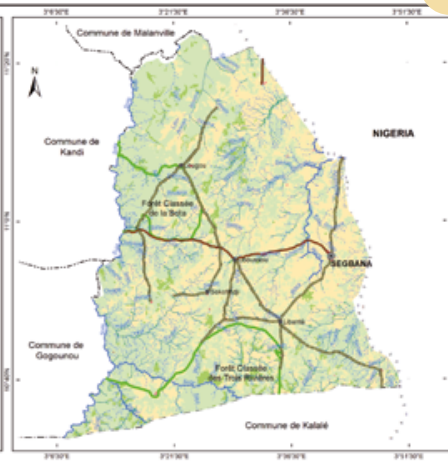

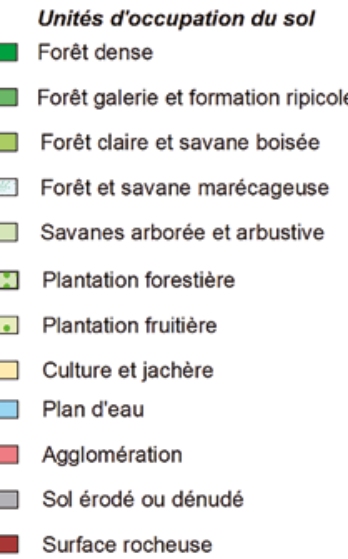

\begin{tabular}{llll}
0 & 8 & 16 & 24 \\
\hline \hline
\end{tabular}

- Chef-lieu d'Arrondissement Limites administratives

++ Limite d'Etat

- Limite de département

-... Limite de commune

- Limite de domaine classé Réseau routier

- Route bitumée

- Route principale

- Route secondaire Réseau hydrographique Cours d'eau permanent Cours d'eau temporaire
Sources: Images SPOT Pivot 2015 (OSFACO) Feuilles topo 1/ 50 000; PAPDFGC, 2015 Données de terrain, 2018 - 2019.

Ellipsoìde WGS 84. Projection UTM

\section{Tableau I.}

Matrice de confusion de l'interprétation visuelle de l'image de 1995 (nombre de points relevés sur l'image et vérifiés sur le terrain).

\begin{tabular}{|c|c|c|c|c|c|c|c|c|c|c|c|c|c|c|c|}
\hline \multirow{2}{*}{$\begin{array}{l}\text { Données relevées } \\
\text { sur le terrain }\end{array}$} & \multirow{2}{*}{ FD } & \multirow[b]{2}{*}{ FGFR } & \multirow{2}{*}{ FCSB } & \multirow[b]{2}{*}{ SASa } & \multirow[b]{2}{*}{ FSM } & \multicolumn{4}{|c|}{ Données interprétées } & \multirow{2}{*}{5 HA } & \multirow[b]{2}{*}{ SR } & \multirow[b]{2}{*}{ SED } & \multirow[b]{2}{*}{ TOTAL } & \multirow[b]{2}{*}{ PU } & \multirow[b]{2}{*}{ Co } \\
\hline & & & & & & PTFR & CJ & PTFT & PE & & & & & & \\
\hline FD & 22 & 2 & 2 & 0 & 0 & 0 & 0 & 0 & 0 & 0 & 0 & 0 & 26 & 85 & 15 \\
\hline FGFR & 0 & 30 & 0 & 0 & 0 & 0 & 0 & 0 & 0 & 0 & 0 & 0 & 30 & 100 & 0 \\
\hline FCSB & 2 & 1 & 80 & 1 & 0 & 0 & 0 & 0 & 0 & 0 & 0 & 0 & 84 & 95 & 5 \\
\hline SASa & 1 & 0 & 5 & 155 & 0 & 3 & 1 & 0 & 0 & 1 & 0 & 0 & 166 & 93 & 7 \\
\hline FSM & 0 & 0 & 2 & 5 & 8 & 0 & 0 & 0 & 0 & 0 & 0 & 0 & 15 & 53 & 47 \\
\hline PTFR & 0 & 0 & 0 & 0 & 0 & 25 & 0 & 2 & 0 & 0 & 0 & 0 & 27 & 93 & 7 \\
\hline CJ & 0 & 0 & 0 & 8 & 0 & 2 & 112 & 2 & 0 & 6 & 0 & 0 & 130 & 86 & 14 \\
\hline PTFT & 0 & 0 & 0 & 0 & 0 & 0 & 0 & 15 & 0 & 0 & 0 & 0 & 15 & 100 & 0 \\
\hline $\mathrm{PE}$ & 0 & 0 & 0 & 0 & 0 & 0 & 0 & 0 & 4 & 0 & 0 & 0 & 4 & 100 & 0 \\
\hline $\mathrm{HA}$ & 0 & 0 & 0 & 1 & 0 & 0 & 4 & 0 & 0 & 55 & 0 & 0 & 60 & 92 & 8 \\
\hline SR & 0 & 0 & 0 & 0 & 0 & 0 & 0 & 0 & 0 & 0 & 5 & 0 & 5 & 100 & 0 \\
\hline SED & 0 & 0 & 0 & 0 & 0 & 0 & 0 & 0 & 0 & 0 & 0 & 8 & 8 & 100 & 0 \\
\hline TOTAL & 25 & 33 & 89 & 170 & 8 & 30 & 117 & 19 & 4 & 62 & 5 & 8 & 570 & & \\
\hline $\mathrm{Pp}(\%)$ & 88 & 91 & 90 & 91 & 100 & 83 & 96 & 79 & 100 & 889 & 100 & 100 & & & \\
\hline $\mathrm{O}(\%)$ & 12 & 9 & 10,11 & 8,82 & 0 & 16,67 & 4,27 & 2,1 & 0 & 11,29 & 0 & 0 & & & \\
\hline
\end{tabular}

PGI : précision globale ; Pp : précision producteur ; 0 : erreur d'omission ; IC : intervalle de confiance ; PU : précision utilisateur ; Co : erreur de commission ; FD : forêt dense ; FGFR : forêt-galerie et formation ripicole ; FCSB : forêt claire et savane boisée ; FSM : forêt et savane marécageuse ; SASa : savane arborée et arbustive ; PTFR : plantation fruitière ; $\mathrm{CJ}$ : champs et jachères ; PTFT : plantation forestière ; PE : plan d'eau ; HA : habitation ; SR : surface rocheuse ; SED : sol dénudé. 
Bois et Forêts des Tropiques - ISSN: L-0006-579X

Volume $346-4^{\text {th }}$ quarter - December 2020 - p. 35-50

42 Focus / LAND VULNERABILITY

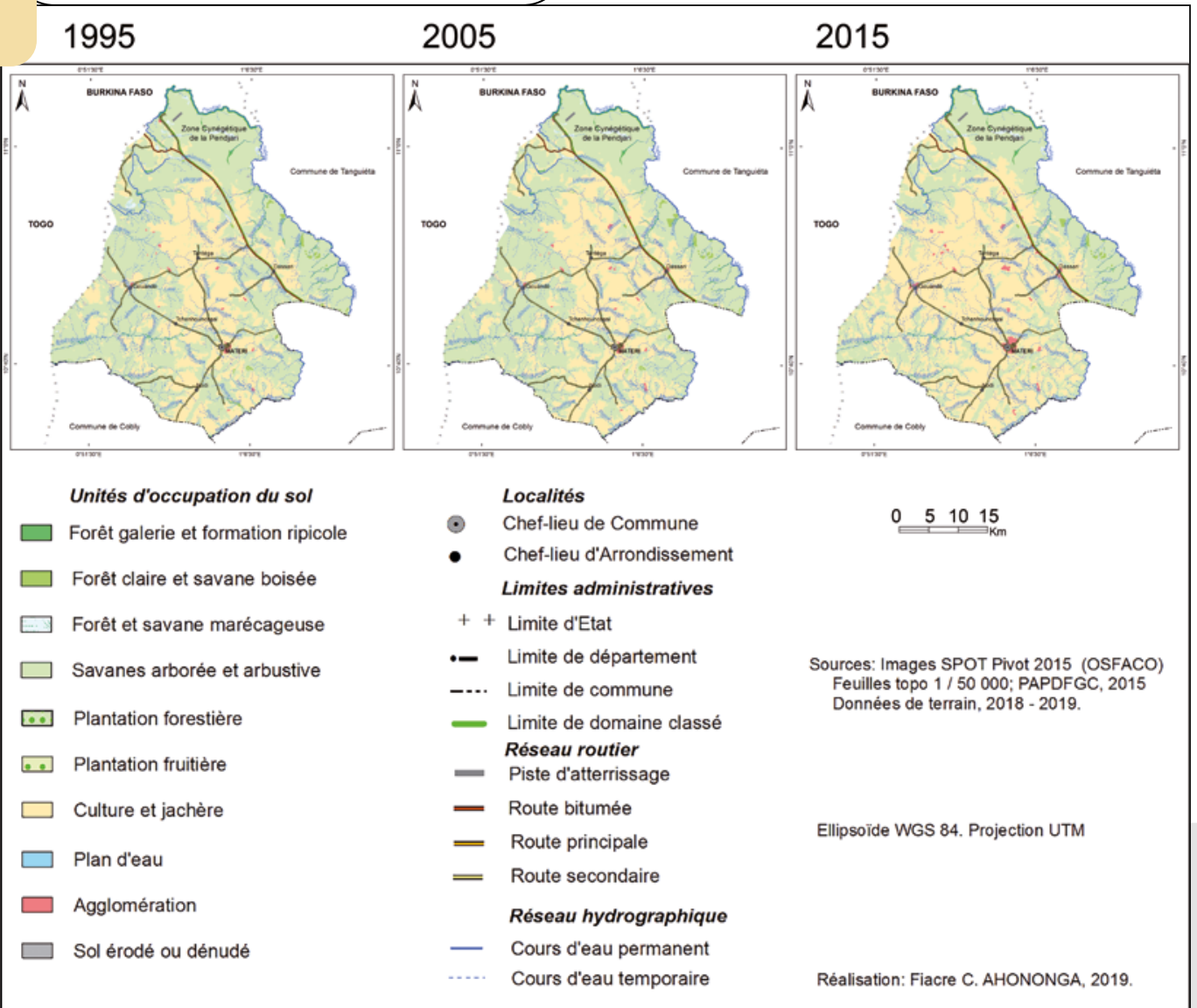

montrent une faible erreur de commission et d'omission. Ainsi, l'interprétation a permis de différencier douze classes d'occupation du sol. Ces classes sont représentées par les forêts (dense, galerie, claire) et les savanes (marécageuse, arborée, arbustive et herbeuse), les champs et jachères, les habitations, les plantations (forestière et fruitière), les plans d'eau, les sols dénudés et les sols rocheux.

\section{Dynamique de l'occupation des terres de 1995 à 2015}

Les résultats des analyses et des cartes montrent que les écosystèmes, qu'ils soient hors ou dans le

Figure 3.

Cartes de l'occupation du sol des écosystèmes de Matéri en 1995 , 2005 et 2015.

Tableau II.

Matrice de confusion de l'interprétation visuelle de l'image de 2005 (nombre de points relevés sur l'image et vérifiés sur le terrain).

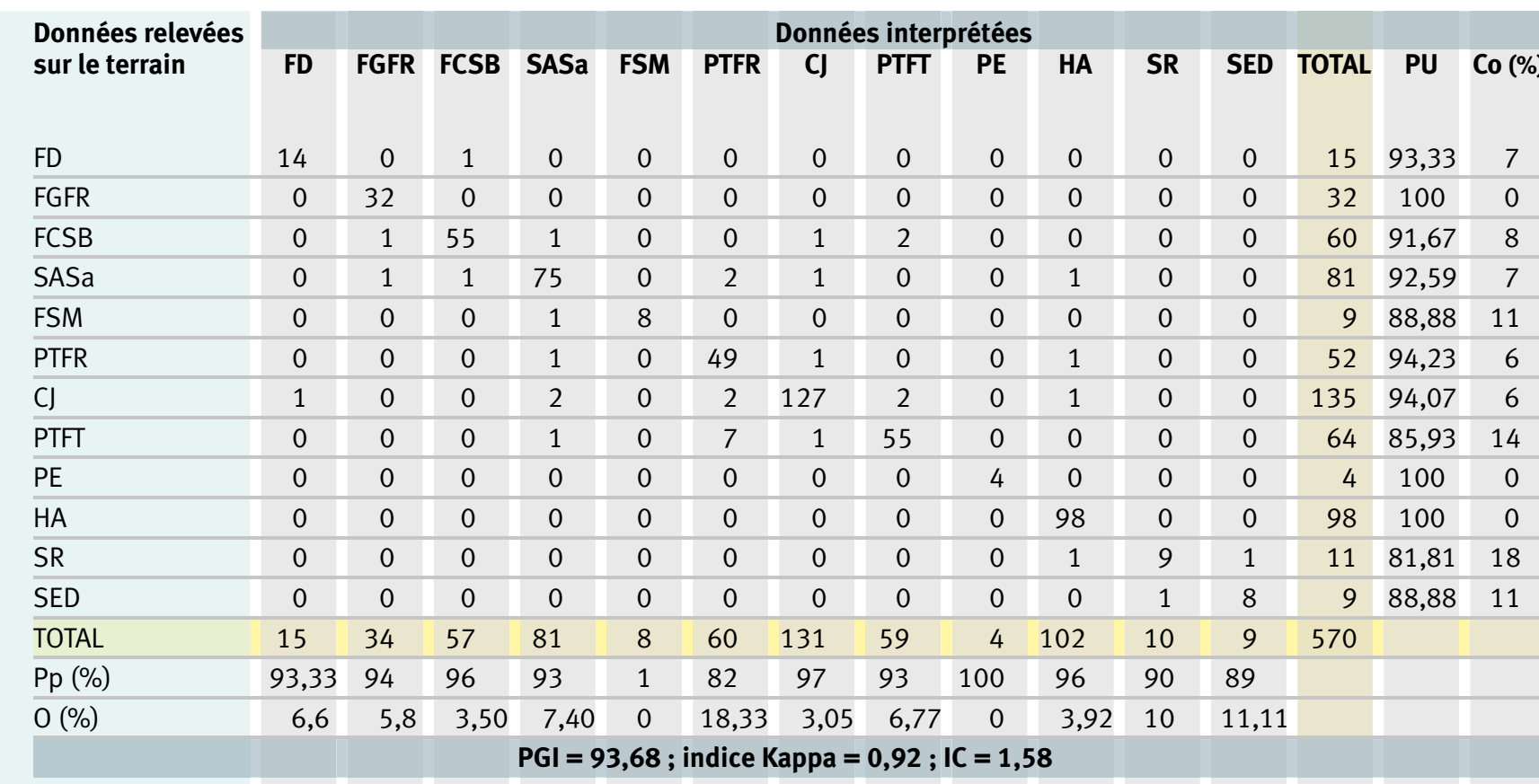

PGI : précision globale ; Pp : précision producteur ; 0 : erreur d’omission ; IC : intervalle de confiance ; PU : précision utilisateur ; Co : erreur de commission ; FD : forêt dense ; FGFR : forêt-galerie et formation ripicole ; FCSB : forêt claire et savane boisée ; FSM : forêt et savane marécageuse ; SASa : savane arborée et arbustive ; PTFR : plantation fruitière ; $\mathrm{CJ}$ : champs et jachères ; PTFT : plantation forestière ; PE : plan d'eau ; HA : habitation ; SR : surface rocheuse ; SED : sol dénudé. 


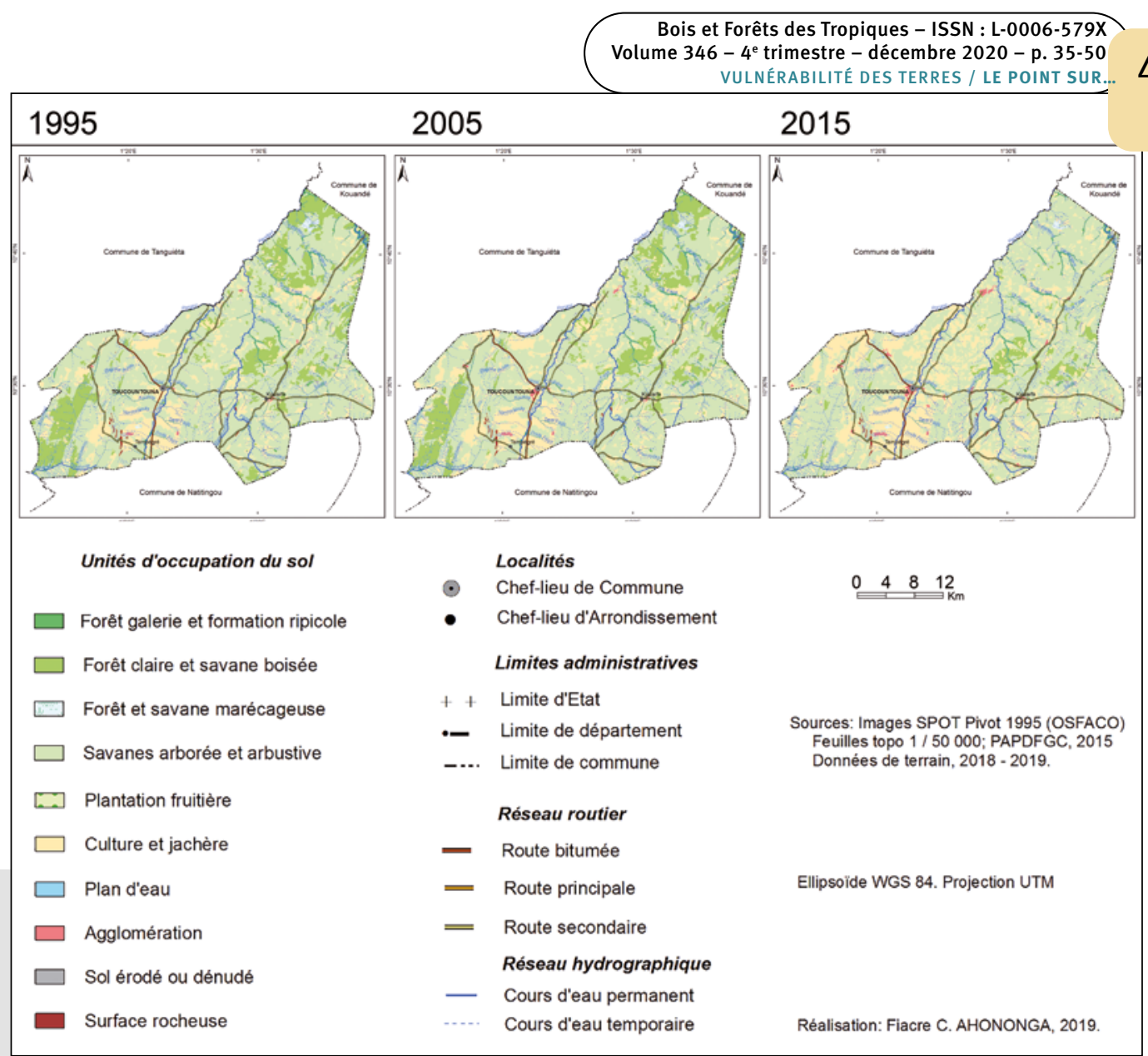

Tableau III.

Matrice de confusion de l'interprétation visuelle de l'image de 2015 (nombre de points relevés sur l'image et vérifiés sur le terrain).

\begin{tabular}{|c|c|c|c|c|c|c|c|c|c|c|c|c|c|c|c|}
\hline \multirow{2}{*}{$\begin{array}{l}\text { Données relevées } \\
\text { sur le terrain }\end{array}$} & \multirow[b]{2}{*}{ FD } & \multirow[b]{2}{*}{ FGFR } & \multirow[b]{2}{*}{ FCSB } & \multirow[b]{2}{*}{ SASa } & \multirow[b]{2}{*}{ FSM } & \multirow[b]{2}{*}{ PTFR } & \multicolumn{4}{|c|}{ Données interprétées } & \multirow[b]{2}{*}{ SR } & \multirow[b]{2}{*}{ SED } & \multirow[b]{2}{*}{ Total } & \multirow[b]{2}{*}{ PU } & \multirow[b]{2}{*}{ Co } \\
\hline & & & & & & & CJ & PTFT & PE & HA & & & & & \\
\hline FD & 14 & 0 & 1 & 1 & 0 & 0 & 0 & 0 & 0 & 0 & 0 & 0 & 16 & 88 & 13 \\
\hline FGFR & 0 & 32 & 0 & 0 & 0 & 0 & 0 & 0 & 0 & 0 & 0 & 0 & 32 & 100 & 0 \\
\hline FCSB & 0 & 1 & 55 & 1 & 0 & 0 & 1 & 2 & 0 & 0 & 0 & 0 & 60 & 92 & 8 \\
\hline SASa & 0 & 1 & 1 & 83 & 0 & 2 & 1 & 0 & 0 & 1 & 0 & 0 & 89 & 93 & 7 \\
\hline FSM & 0 & 0 & 0 & 0 & 11 & 0 & 0 & 0 & 0 & 0 & 0 & 0 & 11 & 100 & 0 \\
\hline PTFR & 0 & 0 & 0 & 1 & 0 & 77 & 1 & 0 & 0 & 1 & 0 & 0 & 80 & 96 & 4 \\
\hline CJ & 1 & 0 & 0 & 1 & 0 & 2 & 150 & 1 & 0 & 0 & 0 & 0 & 155 & 97 & 3 \\
\hline PTFT & 0 & 0 & 0 & 1 & 0 & 2 & 1 & 49 & 0 & 0 & 0 & 0 & 53 & 92 & 8 \\
\hline PE & 0 & 0 & 0 & 0 & 0 & 0 & 0 & 0 & 4 & 0 & 0 & 0 & 4 & 100 & 0 \\
\hline $\mathrm{HA}$ & 0 & 0 & 0 & 0 & 0 & 0 & 0 & 0 & 0 & 97 & 0 & 0 & 97 & 100 & 0 \\
\hline SR & 0 & 0 & 0 & 0 & 0 & 0 & 0 & 0 & 0 & 0 & 7 & 0 & 7 & 100 & 0 \\
\hline SED & 0 & 0 & 0 & 0 & 0 & 0 & 0 & 0 & 0 & 0 & 0 & 4 & 4 & 100 & 0 \\
\hline Total & 15 & 34 & 57 & 88 & 11 & 83 & 154 & 52 & 4 & 99 & 7 & 4 & 608 & & \\
\hline $\mathrm{Pp}$ & 93 & 94 & 96 & 94 & 100 & 93 & 97 & 94 & 100 & 98 & 100 & 100 & & & \\
\hline O (\%) & 7 & 6 & 4 & 6 & 0 & 7 & 3 & 6 & 0 & 2 & 0 & 0 & & & \\
\hline & & & & $\mathrm{GI}=9$ & $\%$; in & lice de & Kарр & 0,95 & $\mathbf{I C}=1$ & & & & & & \\
\hline
\end{tabular}

PGI : précision globale ; Pp : précision producteur ; 0 : erreur d'omission ; IC : intervalle de confiance ; PU : précision utilisateur ; Co : erreur de commission; FD : forêt dense ; FGFR : forêt-galerie et formation ripicole ; FCSB : forêt claire et savane boisée ; FSM : forêt et savane marécageuse ; SASa : savane arborée et arbustive ; PTFR : plantation fruitière ; $\mathrm{Cl}$ : champs et jachères ; PTFT : plantation forestière ; PE : plan d'eau ; HA : habitation ; SR : surface rocheuse ; SED : sol dénudé. 
domaine non classé de Matéri (9,19\%), de Toucountouna $(10,66 \%)$ et de Ségbana (36,02 \%), les champs et jachères ont augmenté en superficie. Dans le domaine non classé, le fait le plus marquant est l'urbanisation, car les superficies des habitations ont augmenté successivement de 30,71\%, $80,65 \%$ et $9,81 \%$.

Au cours de la période de 2005 à 2015, il y a une expansion significative des habitations dans le domaine non classé de Matéri (80,51\%), de Toucountouna (80,65\%) et de Ségbana (171,84\%). De la même façon, les superficies des champs et jachères ont augmenté respectivement de $50,75 \%, 60,23 \%$ et $165,77 \%$ dans le domaine non classé (figures $5 \mathrm{abc}$ ). La régression des superficies des forêts claires et savanes boisées est plus marquée dans la zone de Matéri $(75,66 \%)$ et celle de Toucountouna $(72,34 \%)$. Quant aux forêts denses, elles ont connu une régression de $96,27 \%$ dans le domaine non classé de Matéri (figure 5a). II ressort également que les plantations fruitières ont augmenté au cours de cette période dans le domaine non classé (figures 2, 3 et 4). Elles représentent 102,18 \% de la superficie dans la zone de Toucountouna et $11,11 \%$ dans celle de Matéri. Elles sont quasi absentes dans le domaine classé. Par contre, les plantations forestières ont été prédominantes seulement dans le domaine non classé de Toucountouna $(104,53 \%)$ (figure $5 \mathrm{~b}$ ) alors que dans la même période elles ont diminué à Matéri (- 68,02\%) (figure 5a) et sont quasi absentes dans le domaine classé (figures $5 \mathrm{~d}$, $5 e)$.
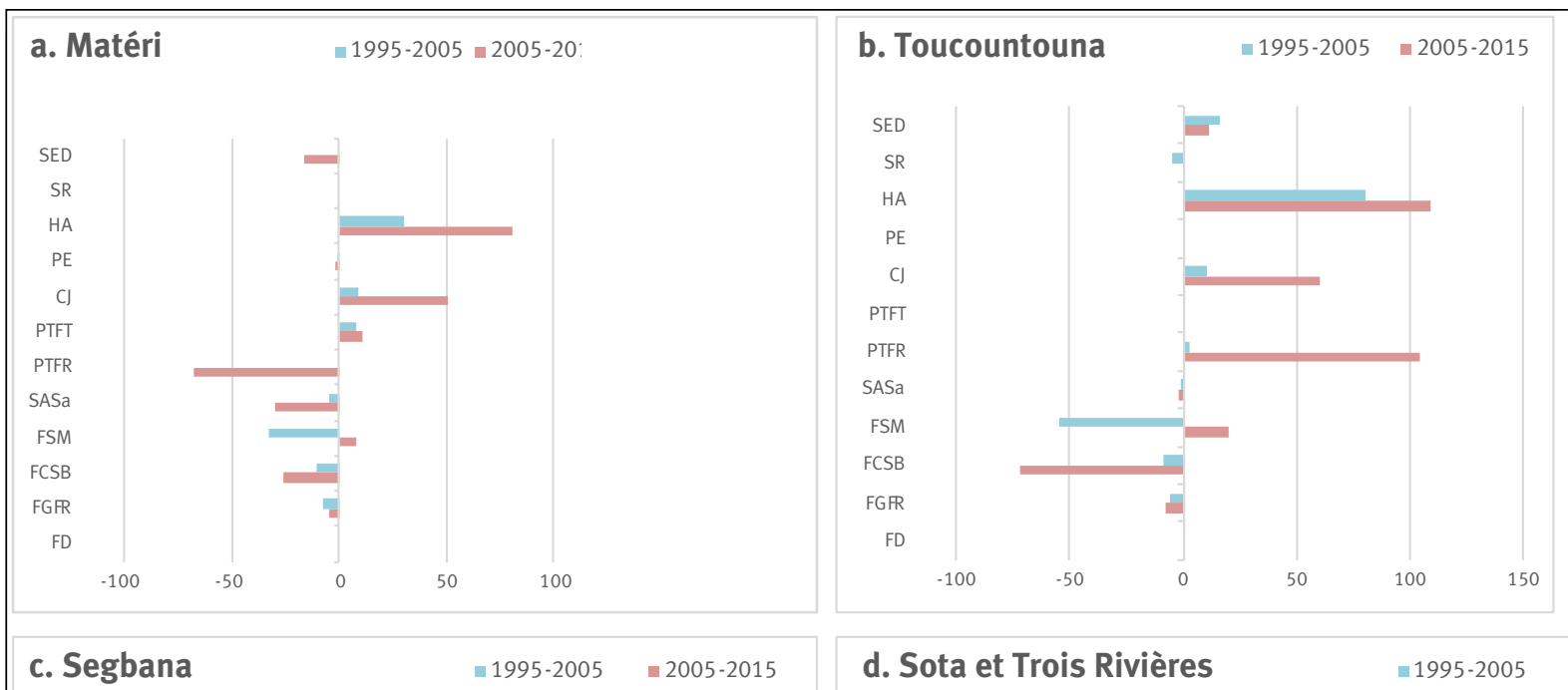

\section{d. Sota et Trois Rivières $\quad$ - $1995-2005-2015$}
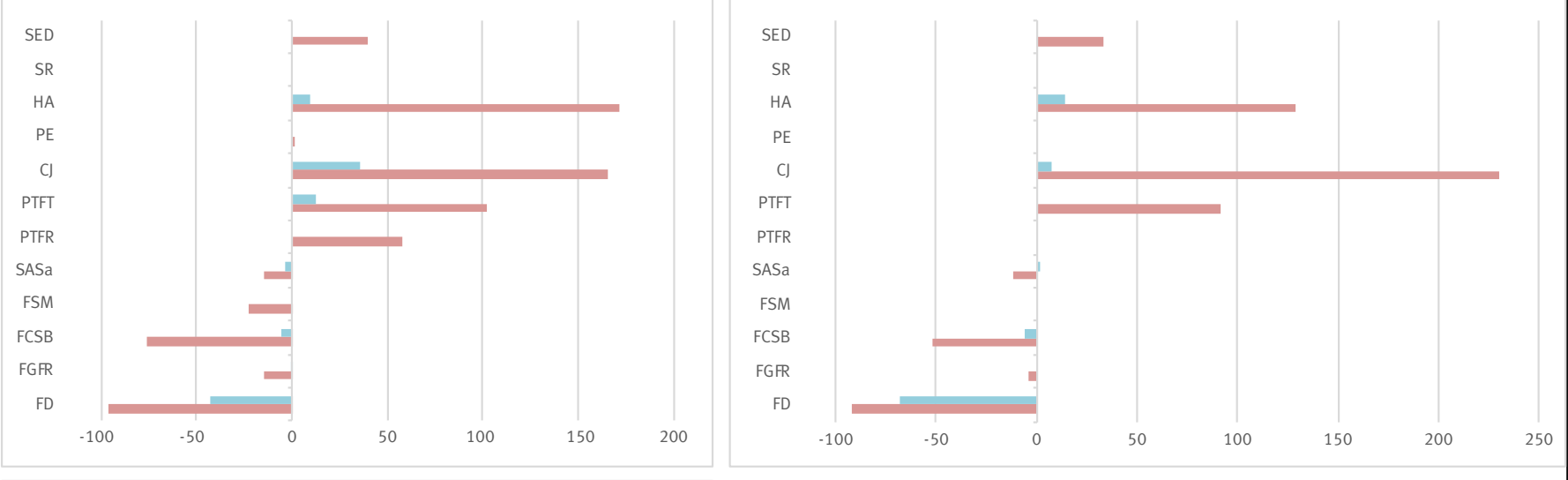

\section{e. ZC Pendjari}

- 1995-2005 - 2005-2015

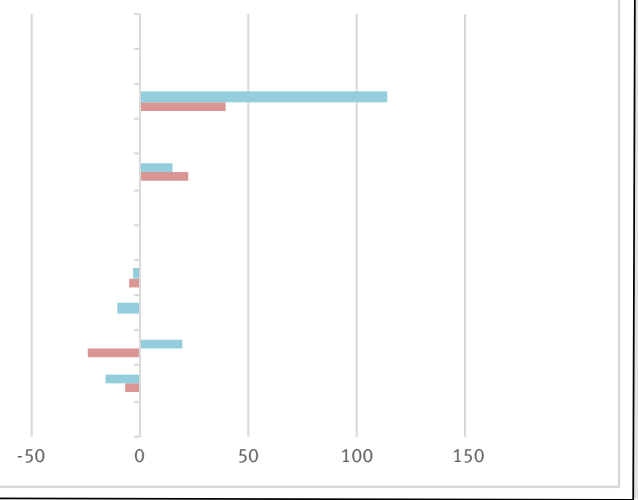

Figure 5 .

Taux d'évolution des unités d'occupation du sol dans la zone soudanienne pour les périodes 1995-2005 et 2005-2015. FD : forêt dense ; FGFR : forêt-galerie et formation ripicole ; FCSB : forêt claire et savane boisée ; FSM : forêt et savane marécageuse ; SASa : savane arborée et arbustive ; PTFR : plantation fruitière ; $\mathrm{CJ}$ : champs et jachères; PTFT : plantation forestière; $P E$ : plan d'eau; HA : habitation; SR : surface rocheuse ; SED : sol dénudé ; ZC : zone cynégétique. 


\section{Effet de la protection et du type de formation sur la dynamique d'affectation des terres}

Le taux d'évolution des différentes formations est déterminé par la période considérée et le niveau de protection. Le taux d'évolution moyen des différents types de formations dans le domaine non classé n'est pas significativement différent entre 1995 et 2005 (figure 6a). Par contre, dans le domaine classé, les taux d'évolution des formations naturelles, non naturelles et des plantations sont significativement différents (figure 6c). Par ailleurs, quel que soit le niveau de protection, les formations naturelles sont régressives. Entre 2005 et 2015, les taux d'évolution des domaines classé et non classé sont significativement différents (figures 6b et $6 \mathrm{~d}$ ). Les formations naturelles ont connu une régression de leur superficie avec un taux plus accentué dans le domaine classé (figure $6 \mathrm{~d}$ ). Au cours de cette période, le domaine classé a connu une expansion des formations non naturelles supérieure à celle du domaine non classé (figures $6 \mathrm{~b}$ et $6 \mathrm{~d}$ ). Également, les taux de reconstitution (plantations) dans le domaine non classé ont connu une progression inférieure à celle du domaine classé (figures $6 \mathrm{~b}$ et $6 \mathrm{~d}$ ).

\section{Effet de la protection et du type de formation sur la vulnérabilité des écosystèmes}

Les analyses montrent que la vulnérabilité au changement des différents types de formations est significativement différente (figure 7) en fonction de la période. Les formations non forestières et les plantations forestières sont plus vulnérables à l'expansion alors que les formations naturelles ont une tendance à la régression. Cette tendance est significativement différente suivant le niveau de protection (figures $7 \mathrm{a}$ et 7c). Par ailleurs, au cours de la période 1995-2005, le domaine non classé a été globalement vulnérable à l'expansion des formations non naturelles alors que le domaine classé était susceptible d'une perte de superficie des formations naturelles (figure $7 a$ ). Au cours de la période 2005-2015, le domaine non classé a été plus vulnérable au changement alors que le domaine classé est resté stable (figures $7 b$ et $7 d$ ).

\section{Analyse de la vulnérabilité et intensité de changement des unités d'occupation du sol et des différentes formations des écosystèmes}

Les taux de vulnérabilité, en perte ou en gain, ont été très variables selon les unités d'occupation des terres et fonction du niveau de protection de celles-ci. Ainsi, entre 1995 et 2005 , dans le domaine non classé, les formations naturelles en général ont connu un gain de superficie plus que de perte (figure 8). Seules les forêts claires ont été faiblement vulnérables à la perte de superficie (figure 8). La même tendance s'est observée dans le domaine classé au cours de cette période. Sur période 2005-2015, les (a)

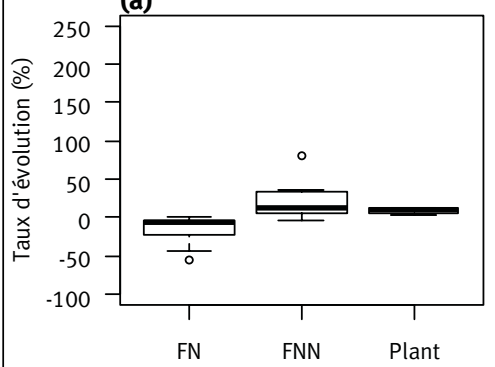

Type de formation

(c)

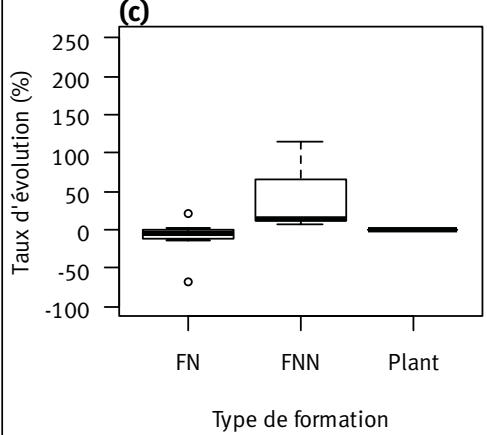

(b)

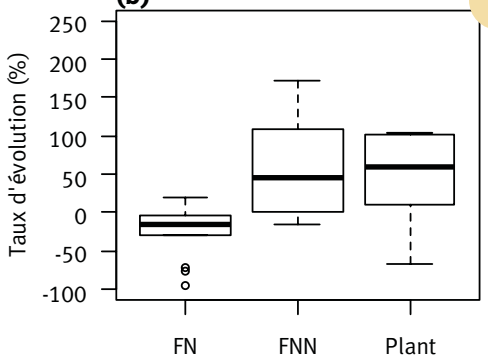

Type de formation

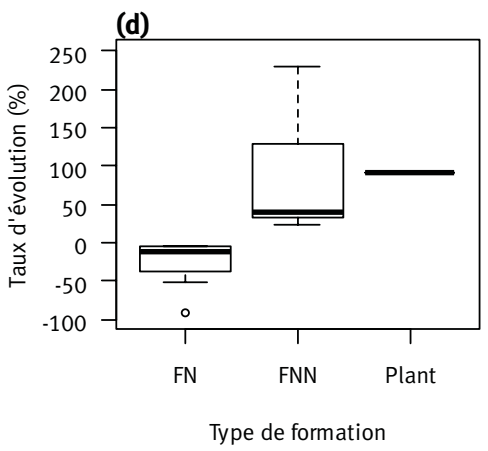

Figure 6.

Effet de la protection (a et c) et du type de formation (b et d) sur le taux d'évolution du couvert végétal. FN : formation naturelle; FNF : formation non forestière ; Plant : plantation forestière.

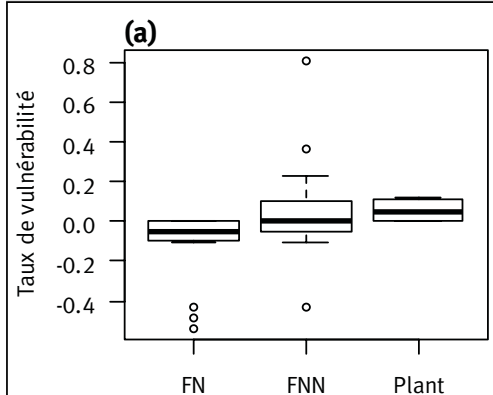

(c)

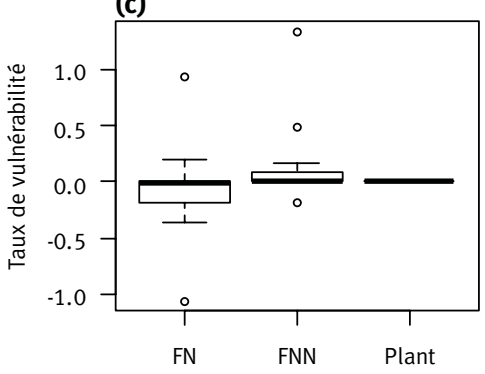

Type de formation
Type de formation (b)

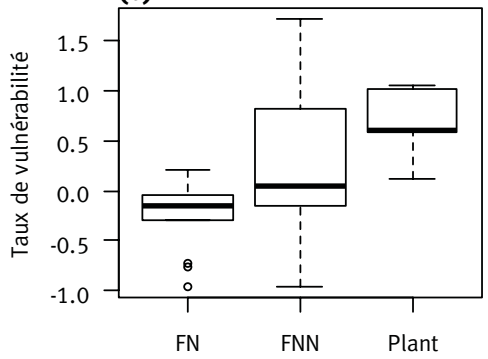

Type de formation

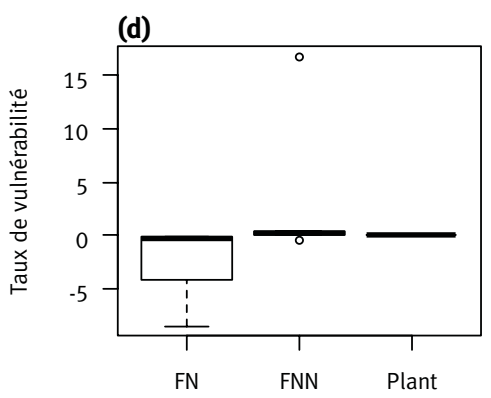

Type de formation
Figure 7.

Intensité des changements et de la vulnérabilité par catégorie d'occupation des terres selon le gradient de protection (a et c) et le type de formation (b et d) au cours des périodes 1995-2005 et 2005-2015. FN : formation naturelle ; FNF : formation non forestière ; Plant : plantation forestière. 
Bois et Forêts des Tropiques - ISSN: L-0006-579X

Volume $346-4^{\text {th }}$ quarter - December 2020 - p. 35-50

46 FOCUS / LAND VULNERABILITY

\section{a. 1995-2005}

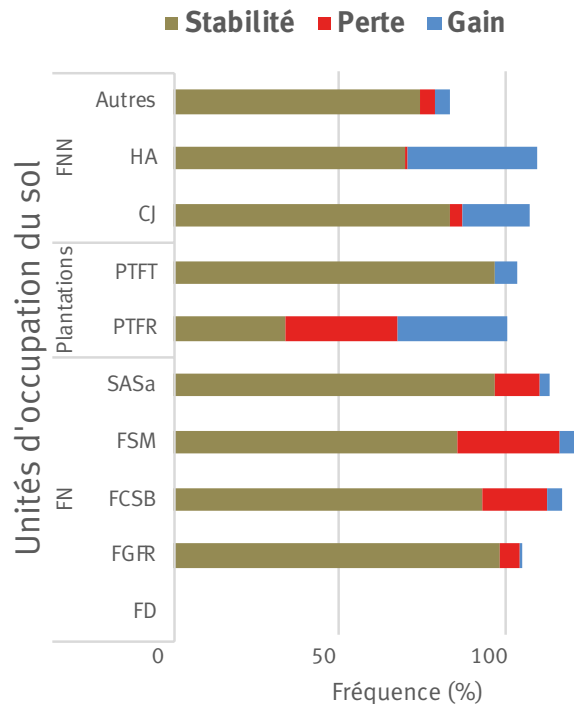

c. 1995-2005

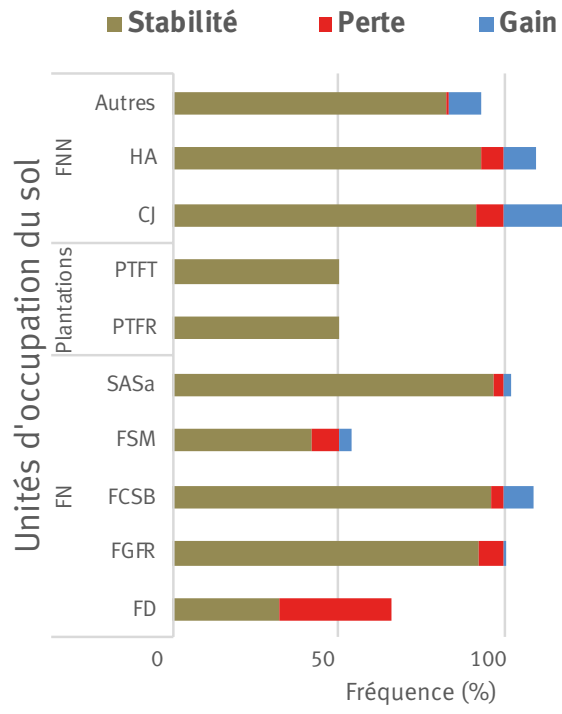

b. 2005-2015

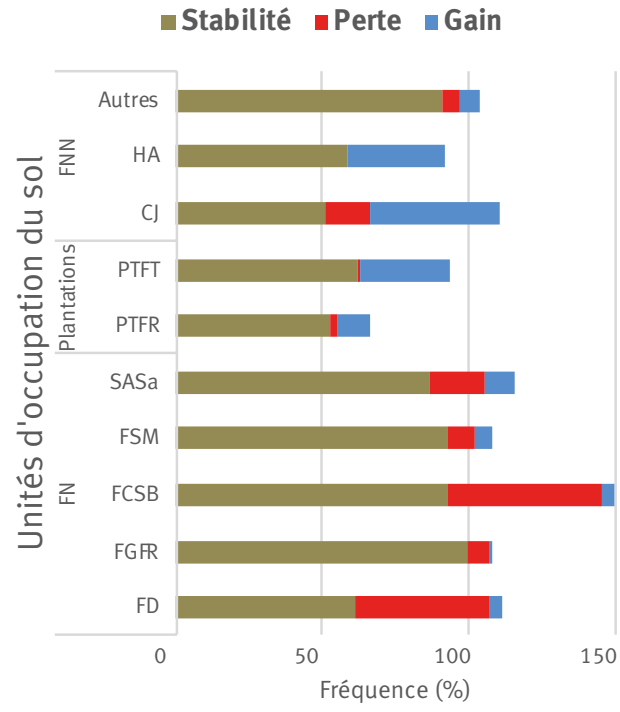

d. 2005-2015

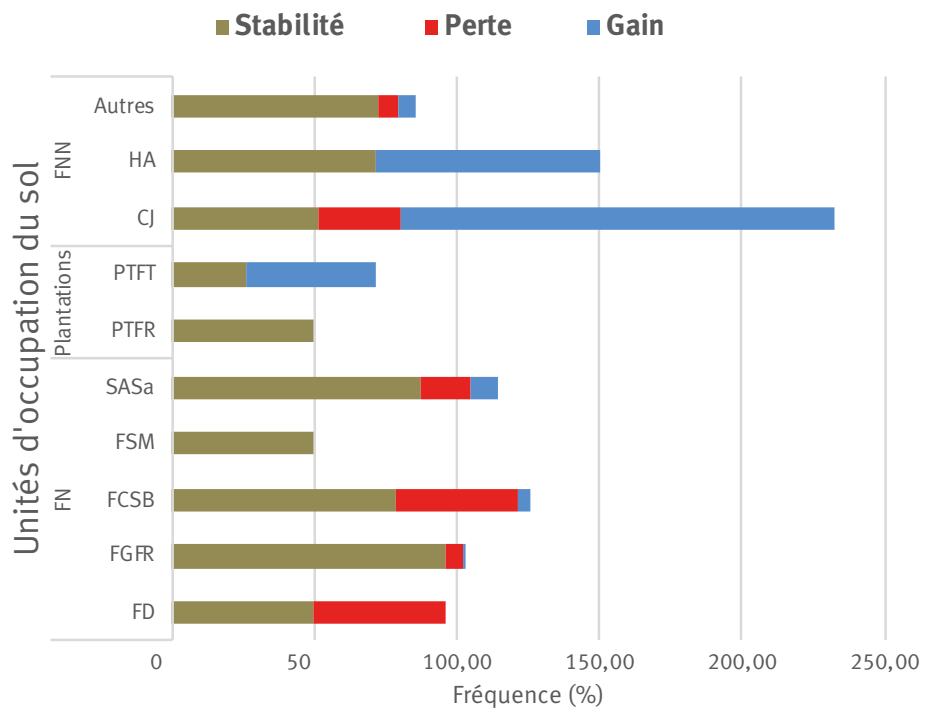

Figure 8.

Intensité des changements et de la vulnérabilité par catégorie d'occupation des terres selon le statut de la zone, domaine non classé ( $a$ et b) et domaine classé (c et d), au cours des périodes 1995-2005 et 2005-2015. FD : forêt dense ; FGFR : forêt-galerie et formation ripicole ; FCSB : forêt claire et savane boisée ; FSM : forêt et savane marécageuse ; SASa : savane arborée et arbustive ;

PTFR : plantation fruitière ; CJ : champs et jachères ; PTFT : plantation forestière ; HA : habitation ; FN : formation naturelle ;

FNF : formation non forestière ; Plant : plantation forestière.

savanes arborées et arbustives ont diminué au profit des champs et jachères où le taux de gain est élevé. La situation est plus alarmante dans le domaine classé, car les savanes arborées et arbustives et les forêts claires ont été très vulnérables à la perte de superficie contrairement aux formations non naturelles (champs et habitations) (figure 8).

\section{Analyse de la déforestation et de la dégradation des écosystèmes forestiers}

Le taux de déforestation diminue avec le niveau de protection alors que le taux de dégradation augmente (figure 9b). Le taux annuel de la déforestation est passé de 0,37\% à 2,33\% entre les périodes 1995-2005 et 2005-2015 dans le domaine non classé alors que dans le domaine classé il 
est respectivement égal à 0,24\% et 0,91\% (figure 9b). Le taux de dégradation a été estimé à 3,27\% dans le domaine non classé et à 13,28 \% dans le domaine classé au cours de la période 2005-2015 (figure 9b).

Les taux de dégradation et de déforestation varient donc en fonction du type de domaine. Dans la commune de Matéri, il ressort qu'au cours de la période 1995 à 2005 le domaine non classé $(0,5 \% / a n)$ est plus affecté par la déforestation et la dégradation que le domaine classé représenté par la zone cynégétique de la Pendjari $(0,43 \% / a n)$ (figure $9 a)$. En revanche, entre 2005 et 2015, la déforestation s'est arrêtée dans le domaine classé, mais la dégradation s'est accentuée avec un taux annuel de $2 \%$. Cette dégradation est absente dans le domaine non classé (figure 9a).

En ce qui concerne la commune de Ségbana, la dégradation est plus accentuée dans le domaine classé ( $24 \%$ et $2 \%$ respectivement au cours de la période 2005 2015 et 1995-2015) que dans les écosystèmes du domaine non classé (5\% et $1 \%$ respectivement au cours de la période 2005-2015 et 1995-2015) (figure 9a).

Dans la commune de Toucountouna, il ressort qu'entre les périodes 1995-2005 et 2005-2015 les taux annuels de déforestation nette sont respectivement de $0,2 \%$ et $1,4 \%$ dans les écosystèmes du domaine non classé. Au cours de ces périodes, les taux de dégradation nette des formations forestières sont respectivement de $1 \%$ et $5 \%$ entre 1995 2005 et 2005-2015 (figure 9a).

\section{Discussion}

\section{Approche méthodologique}

Il est évident qu'une opération d'interprétation visuelle est liée à l'expérience de l'opérateur. Ainsi, l'interprétation visuelle des non-experts est un processus plus intuitif alors que les spécialistes procèdent logiquement et étape par étape. Dans cette étude, pour réduire l'effet de l'interprétation intuitive, les missions de reconnaissance et de contrôle terrain ont été renforcées. Aussi, la résolution spatiale assez fine des images utilisées a permis d'affiner la qualité de l'interprétation, comme l'illustrent les valeurs du coefficient de Kappa acceptables des images des différentes années considérées. Bien que l'interprétation visuelle fournisse des résultats acceptables, ses limites fondamentales sont le coût et le temps de sa réalisation, contrairement à l'utilisation des algorithmes de classification automatique qui peut induire des confusions spectrales. Par conséquent, la combinaison des deux méthodes permet de réduire certaines difficultés (coûts, temps et confusions spectrales) pour obtenir un meilleur résultat.
Figure 9.

Taux de déforestation et de dégradation du couvert forestier suivant le gradient de protection (a) et e domaine (b) au cours des périodes 1995-2005 et 2005-2015. ZC : zone cynégétique ; Tctna : Toucountouna ; Ségba : Ségbana ; FC : forêt classée.

\section{Déforestation et dégradation des différents écosystèmes du domaine soudanien}

Les changements d'occupation et d'utilisation des terres dans les écosystèmes de la zone soudanienne révèlent une régression des formations forestières naturelles au profit des formations non forestières (champs, jachères, habitations) aussi bien dans le domaine non classé que dans le domaine classé. En 1995, l'occupation des sols de la zone était dominée par les formations naturelles, mais en 2005, elle est dominée par les cultures, les jachères et les habitations. Au cours de la période 2005-2015, les formations naturelles ont donc laissé progressivement place aux formations anthropiques. Des résultats similaires sont obtenus par Ousseni et al. (2016) et Avakoudjo et al. (2015) respectivement dans le bassin supérieur de l'Alibori et le parc W au Bénin, et confirment les résultats de la FAO (2010) qui font état de la réduction des superficies forestières entre 1990 et 2010 . Il en a été de même pour Mamane et al. (2018) dans la Réserve totale de faune de Tamou au Niger.

Néanmoins, nos résultats montrent que l'ampleur du phénomène est fonction du degré de protection de ces écosystèmes. Ainsi, la déforestation est plus prononcée dans le domaine non classé que dans le domaine classé. À l'inverse, les domaines classés de la Sota et des Trois Rivières ont été 
plus affectés par la dégradation que le domaine non classé. Ce phénomène peut s'expliquer par la nature différente des processus anthropiques à l'œuvre dans les deux zones.

Dans le domaine non classé, le processus principal menant à la déforestation est l'intensification des activités anthropiques (Kpedenou et al., 2016) : expansion de l'agriculture, installation de nouvelles habitations et exploitation du bois. Ces facteurs sont par ailleurs rapportés par de nombreux auteurs (Geist et Lambin, 2002 ; Barima et al., 2009 ; Ouedraogo et al., 2010 ; Damnyag et al., 2013 ; Ahmad et Pandey, 2018 ; Sikuzani et al., 2019).

Dans le domaine classé, on observe également une part de déforestation. Nos résultats corroborent ainsi les observations faites par Houéssou et al. (2013), Avakoudjo et al. (2015) et Mamane et al. (2018) et confirment les pressions que subissent les aires protégées de la part des populations périphériques. En particulier, pendant ces dix dernières années (2005 à 2015), les trois aires classées ont connu un développement important des nouvelles habitations et des nouveaux champs. Ce non-respect des règles et des limites du domaine classé pourrait être dû à plusieurs facteurs : la méconnaissance ou l'ignorance des limites de celui-ci par la population locale (Temgoua et al., 2018) ; le non-respect des pratiques ancestrales de conservation de la nature par les jeunes générations (Mama et al., 2013) ; le développement de la filière coton et de certaines spéculations pionnières comme l'igname qui entraîne l'installation de nouveaux exploitants ; et, enfin, l'abandon de terres devenues infertiles dans les zones périphériques au profit de terres encore fertiles dans les forêts classés. La progression des sols nus dans le domaine non classé est en effet un indicateur de perte de fertilité. Le même constat a été fait au Maroc par Rifai et al. (2018), qui expliquent le phénomène par les besoins de terres de construction et une perte de fertilité des sols.

Mais on observe également une dégradation des massifs forestiers dont l'origine pourrait être multiple. Selon certains auteurs, les forêts classées seraient plus affectées par l'exploitation forestière sélective qui diminue la densité du couvert forestier (Adjonou et al., 2010). Pour d'autres auteurs, l'élevage transhumant au travers du piétinement et du passage des troupeaux pourrait être également une cause de la diminution de la régénération des arbres, affectant alors la composition du couvert forestier (Bouko et al., 2016; Amahowe et al., 2018).

Dans tous les cas, la faible surveillance des forêts classées engendre les infractions aux règles de préservation des milieux et laisse libre cours aux activités humaines dégradant les écosystèmes forestiers (Ousmane et al., 2020). De pareils constats sont également faits par Arouna et al. (2017) dans les forêts classées de l'Alibori-Supérieur, mais aussi par Biaou et al. (2019) dans la forêt classée de Ouénou-Bénou. Ces forêts classées sont dans les mêmes districts que les forêts classées des Trois Rivières et de la Sota.

Par ailleurs, les actions de reconstitution ont été plus fortes dans le domaine non classé que dans le domaine classé. Ces actions sont pour la plupart menées avec des espèces exotiques fruitières. Les plantations fruitières, en particulier l'anacardier, occupent toujours le premier rang pour les communautés locales compte tenu de leur importance dans l'amélioration des revenus. À Toucountouna, où le taux de défores- tation annuel est de 1,4\% avec un taux annuel de dégradation de $5 \%$, les plantations qu'elles soient forestières ou fruitières sont quasi absentes. Une politique de sensibilisation et de formation sur les techniques de reboisement des espèces endogènes importantes pour le bien-être socio-économique de la population serait un moyen pour initier une dynamique de replantation.

De la même manière, les actions de reforestation sont actuellement très limitées dans les zones classées (aucune n'est en cours dans la Pendjari) et demanderaient à être intensifiées pour diminuer la dégradation.

\section{Vulnérabilité des écosystèmes}

Dans les forêts classées de la Sota et des Trois Rivières, ce sont les forêts-galeries et les savanes qui ont été les plus vulnérables entre 2005 et 2015. La vulnérabilité des forêts claires, forêts denses, savanes et forêts-galeries pourrait s'expliquer essentiellement par les défrichements en vue de l'installation de nouveaux champs (Rifai et al., 2018). La vulnérabilité de ces formations naturelles qui regorgent d'une forte diversité d'espèces constitue une menace pour la conservation de la biodiversité et des habitats. Les résultats de Bouko et al. (2007) ont montré l'impact négatif de l'expansion agricole sur la composition des communautés d'espèces : la pression des activités agricoles sur les formations forestières naturelles diminue de manière significative la richesse spécifique de la flore ligneuse, et désorganise la structure naturelle des peuplements (Bouko et al., 2007). Cette désorganisation de la structure naturelle conduit à une fragmentation reconnue comme étant une menace majeure pour la biodiversité à l'échelle mondiale (Fahrig, 2002 ; Yeo et al., 2013). Réduire la perte de la biodiversité nécessite donc la mise en place d'une politique de gestion efficace du sol et un aménagement du territoire.

\section{Conclusion}

Aujourd'hui, la demande de plus en plus forte en terres cultivables fertiles et la croissance démographique ont entrấné une nette régression des écosystèmes forestiers, une situation aussi bien présente dans le domaine non classé que dans les réserves forestières destinées à la conservation de la biodiversité. Les phénomènes de déforestation et de dégradation des milieux forestiers constituent une menace majeure pour la biodiversité. Ces faits observés suscitent l'urgence de la prise de mesures de gestion et de restauration de ces écosystèmes. Les gestionnaires devront se baser sur la gestion intégrée du territoire qui prend en compte la périphérie des aires classées. Une planification efficace de l'occupation des terres dans la périphérie et l'adoption de techniques de gestion durable des terres, ainsi que la gestion intégrée de la fertilité des sols pouvant réduire la déforestation et la dégradation des écosystèmes forestières, devraient être mises en place.

\section{Remerciements}

Nos remerciements s'adressent au projet Observation spatiale des forêts d'Afrique centrale et de l'Ouest (OSFACO) pour avoir mis à disposition les images SPOT nécessaires à cette étude. 


\section{Reférences}

Adjonou K., Ali N., Kokutse A. D., Kokou K., Novigno S. K., 2010. Étude de la dynamique des peuplements naturels de Pterocarpus erinaceus Poir. (Fabaceae) surexploités au Togo. Bois et Forêts des Tropiques, 306 (4) : 45-56. https://doi.org/10.19182/bft2010.306. a20431

Adomou A. C., 2005. Vegetation patterns and environmental gradients in Benin: Implications for biogeography and conservation. $\mathrm{PhD}$ thesis, Wageningen University, The Netherlands, $150 \mathrm{p}$.

Ahmad N., Pandey P., 2018. Assessment and monitoring of land degradation using geospatial technology in Bathinda district, Punjab, India. Solid Earth, 9: 75-90.

Amahowe O. I., Azihou A. F., Armand N. K., Biaou S. S. H., Séverin B., Dieu-Donné A., et al., 2018. Constraints of seedling survival and recruitment under adult tree of a multi-use species Afzelia africana $\mathrm{Sm}$ and Pers in Sudanian region of Benin (West Africa). Agroforestry Systems, 93: 1147-1156. https://doi.org/10.1007/s10457-0180218-6

Arouna O., Etene C. G., Issiako D., 2017. Dynamique de l'occupation des terres et état de la flore et de la végétation dans le bassin supérieur de l'Alibori au Bénin. Journal of Applied Biosciences, 108 (1) : 10543-10552. https://doi.org/10.4314/jab.v108i1.7

Avakoudjo J., Mama A., Toko I., Kindomihou V., Sinsin B., 2015. Dynamique de l'occupation du sol dans le Parc National du W et sa périphérie au nord-ouest du Bénin. International Journal of Biological and Chemical Sciences, 8 (6) : 2608-2625. https://doi. org/10.4314/ijbcs.v8i6.22

Bah O. A., Kone T., Yaffa S., Ndiaye M. L., 2019. Land Use and Land Cover Dynamics in Central River Region of the Gambia, West Africa from 1984 to 2017. American Journal of Modern Energy, 5 (2): 5-18. https://doi.org/10.11648/i.ajme.20190502.11

Barima Y. S. S., Barbier N., Bamba I., Traoré D., Lejoly J., Bogaert J., 2009. Dynamique paysagère en milieu de transition forêt-savane ivoirienne. Bois et Forêts des Tropiques, 299 (1) : 15-25. https:// doi.org/10.19182/bft2009.299.a20419

Batar A. K., Watanabe T., Kumar A., 2017. Assessment of landuse/land-cover change and forest fragmentation in the Garhwal Himalayan Region of India. Environments, 4 (2): 34. https://doi. org/10.3390/environments4020034

Baudron F., Tomscha S. A., Powell B., Groot J. C. J., Gergel S. E., Sunderland T., 2019. Testing the Various Pathways Linking Forest Cover to Dietary Diversity in Tropical Landscapes. Frontiers in Sustainable Food Systems, 3: 97. https://doi.org/10.3389/ fsufs.2019.00097

Benbrahim K. F., Ismaili M., Benbrahim S. F., Tribak A., 2004. Problèmes de dégradation de l'environnement par la désertification et la déforestation : impact du phénomène au Maroc. Science et Changements Planétaires / Sécheresse, 15 (4) : 307-320. https:// www.jle.com/fr/revues/sec/e-docs/problemes de degradation de lenvironnement par la desertification et la deforestation impact du_phenomene_au_maroc_264713/article.phtml

Biaou S., Houeto F., Gouwakinnou G., Biaou H. S. S., Awessou B., Tovihessi S., Tete R., 2019. Dynamique spatio-temporelle de l'occupation du sol de la forêt classée de Ouénou-Bénou au Nord Bénin. In : Conférence OSFACO : Des images satellites pour la gestion durable des territoires en Afrique, 13-15 mars 2019, Cotonou, Bénin, 20 p. https://hal.archives-ouvertes.fr/hal-02189367

Bouko B., Sinsin B., Goura Soulé B., 2007. Effets de la dynamique d'occupation du sol sur la structure et la diversité floristique des forêts claires et savanes au Bénin. Tropicultura, 25 (4) : 221-227. http://www.tropicultura.org/text/v25n4/221.pdf

Bouko B. S., Dossou P. J., Amadou B., Sinsin B., 2016. Exploitation des ressources biologiques et dynamique de la forêt classée de la Mekrou au Bénin. European Scientific Journal, 12 (36) : 228-244. https://doi.org/10.19044/esj.2016.v12n36p228

Brandt M., Wigneron J.-P., Chave J., Tagesson T., Penuelas J., Ciais P., et al., 2018. Satellite passive microwaves reveal recent climateinduced carbon losses in African drylands. Nature Ecology and
Evolution, 2 (5): 827-835. https://doi.org/10.1038/s41559-0180530-6

Coulibaly L., Kouassi K. H., Soro G. E., Savane I., 2016. Analyse du processus de savanisation du nord de la Côte d'Ivoire par télédétection: Cas du département de Ferkessédougou. International Journal of Innovation and Applied Studies, 17 (1) : 136-143. http:// www.jijas.issr-journals.org/abstract.php?article=IJIAS-16-109-07 Damnyag L., Saastamoinen O., Blay D., Dwomohb F. K., Anglaaere L. C. N., Pappinen A., 2013. Sustaining protected areas: Identifying and controlling deforestation and forest degradation drivers in the Ankasa Conservation Area, Ghana. Biological Conservation, 165: 86-94. https://doi.org/10.1016/i.biocon.2013.05.024

Fahrig L., 2002. Effect of habitat fragmentation on extinction threshold: A synthesis. Ecological Applications, 12 (2): 346-353. https://doi.org/10.1890/1051-0761(2002)012[0346:EOHFOT]2.0 . $\mathrm{CO} ; 2$

FAO, 2010. Global Forest Resources Assessment 2010: Final Report. Rome, Italy, FAO, $163 \mathrm{p}$.

FAO, 2016. Forêts et agriculture : défis et possibilités concernant l'utilisation des terres. Rome, Italie, FAO, $137 \mathrm{p}$.

FAO, 2018. La situation des forêts du monde. Les forêts au service du développement durable. Rome, Italie, FAO, $158 \mathrm{p}$.

Geist H., Lambin E. F., 2002. Proximate causes and underlying driving forces of Tropical deforestation. Bioscience, 52: 143-150. https://doi.org/10.1641/0006-3568(2002)052[0143:PCAUDF]2.0 . $\mathrm{CO} ; 2$

Gouwakinnou G. N., Biaou S., Biaou S. S. H., Houéto F. O., 2019. Effet des changements d'occupation des terres sur le flux du carbone : un exemple des forêts classées de l'Alibori-Supérieur et de OuénouBénou au Bénin. In : Orekan O. A. V., Mertens B., Ahononga C. F., Tente H. A. B. (éds). Images satellitaires pour un meilleur aménagement des territoires et une gestion durable de la biodiversité. Actes de la conférence, 25-26 juillet 2018. Cotonou, Bénin, OSFACO, 153-172. https://www.researchgate.net/publication/337812765_Effet des changements d\%27occupation_des terres sur_le flux du carbone un exemple des forets classees de $1 \% 27$ AliboriSuperieur et de Ouenou-Benou au Benin

Hammi S., Simonneaux V., Alifriqui M., Auclair L., Ontes N., 2007. Évolution des recouvrements forestiers et de l'occupation des sols entre 1964 et 2002 dans la haute vallée des Ait Bouguemez (Haut Atlas central, Maroc). Science et Changements Planétaires / Sécheresse, 18 (4) : 271-277. https://www.jle.com/10.1684/ sec.2007.0101

Houéssou L. G., Tèka O., Imorou I. T., Lykke A. M., Sinsin B., 2013. Land use and land-cover change at W Biosphere Reserve and its surroundings areas in Benin Republic (West Africa). Environment and Natural Resources Research, 3 (2): 87-101. https://doi. org/10.5539/enrr.v3n2p87

Hountondji Y. H., 2008. Dynamique environnementale en zones sahélienne et soudanienne de l'Afrique de l'Ouest: Analyse des modifications et évaluation de la dégradation du couvert végétal. Thèse de doctorat, Université de Liège, Belgique, 153 p.

INSAE, 2016. Cahier des villages et quartiers de ville du département de l'Atacora. Cotonou, Bénin, 36 p.

IPBES, 2019. Rapport de la Plénière de la Plateforme intergouvernementale scientifique et politique sur la biodiversité et les services écosystémiques sur les travaux de sa septième session. Paris, France, Nations unies, 53 p. https://ipbes.net/sites/default/ files/ipbes_7 10_add.1_fr.pdf

Kaboré S. A., 2015. Évaluation des services écosystémiques de Crateva adansonii DC., Sarcocephalus latifolius (Smith) Bruce et Burkea africana Hook. dans la région du Sud-Ouest du Burkina Faso. Thèse de doctorat, Université polytechnique de Bobo-Dioulasso, Burkina Faso, $181 \mathrm{p}$.

Kpedenou K. D., Boukpessi T., Tchamie T. T. K., 2016. Quantification des changements de l'occupation du sol dans la préfecture de Yoto (Sud-Est Togo) à l'aide de l'imagerie satellitaire Landsat. Revue des Sciences de l'Environnement, $13: 137-156$. https://hal.archivesouvertes.fr/hal-01409418/document

Liang Y., Liu L., 2017. Simulating land-use change and its effect on biodiversity conservation in a watershed in northwest China. Ecosystem Health and Sustainability, 3 (5): 1335933. https://doi. org/10.1080/20964129.2017.1335933 
Liu J., Coomes D. A., Gibson L., Hu G., Liu J., Luo Y., et al., 2019. Forest fragmentation in China and its effect on biodiversity. Biological Reviews, 94 (5): 1636-1657. https://doi.org/10.1111/brv.12519 Mama A., Sinsin B., De Cannière C., Bogaert J., 2013. Anthropisation et dynamique des paysages en zone soudanienne au nord du Bénin. Tropicultura, 31 (1) : 78-88. http://www.tropicultura.org/ text/v31n1/78.pdf

Mama V. J., Oloukoi J., 2003. Traitements analogiques des images satellitaires dans l'étude de la dynamique de l'occupation du sol. Télédétection, 3 (5) : 429-441. https://www.researchgate.net/ publication/325120156 EVALUATION DE LA PRECISION DES TRAITEMENTS ANALOGIQUES DES IMAGES SATELLITAIRES DANS L\%27ETUDE_DE_LA_DYNAMIQUE_DE_L\%270CCUPATION DU SOL

Mamane B., Amadou G., Barage M., Comby J., Ambouta J. M. K., 2018. Dynamique spatio-temporelle d'occupation du sol dans la Réserve Totale de Faune de Tamou dans un contexte de la variabilité climatique (Ouest du Niger). International Journal of Biological and Chemical Sciences, 12 (4) : 1667-1687. https://doi.org/10.4314/ iibcs.v12i4.13

Oloukoi J., 2013. Scénario socio-économique et écologique des changements de l'occupation des terres au Bénin. Vertig0, 13 (1) : 13267. https://doi.org/10.4000/vertigo.13267

Ouedraogo I., Tigabu M., Savadogo P., Compaore H., Ode P. C., Ouadba J. M., 2010. Land cover change and its relation with population dynamics in Burkina Faso, West Africa. Land Degradation and Development, 21: 453-462. https://doi.org/10.1002/ldr.981 Ousmane S., N'da Dibi H., Kouassi K. H., Kouassi K. E., Ouattara K., 2020. Crises politico-militaires et dynamique de la végétation du Parc national du Mont Péko en Côte d'Ivoire. Bois et Forêts des Tropiques, 343 : 27-37. https://doi.org/10.19182/bft2020.343. a31837

Ousseni A., Gervais E. C., Dramane I., 2016. Dynamique de l'occupation des terres et état de la flore et de la végétation dans le bassin supérieur de l'Alibori au Bénin. Journal of Applied Biosciences, 108: 10531-10542. https://doi.org/10.4314/iab. v108i1.7

Pareta K., Pareta U., 2011. Forest carbon management using satellite remote sensing techniques: a case study of Sagar district. International Scientific Research Journal, 33 (4): 335-348. http://citeseerx.ist.psu.edu/viewdoc/ download?doi=10.1.1.684.7623\&rep=rep1\&type $=$ df

Polasky S., Nelson E., Pennington D., Johnson K. A., 2011. The Impact of Land-Use Change on Ecosystem Services, Biodiversity and Returns to Landowners: A Case Study in the State of Minnesota. Environmental and Resource Economics, 48: 219-242. https://doi. org/10.1007/s10640-010-9407-0

Quantum GIS, 2013. Development Team. Quantum GIS geographic information system. Open Source Geospatial Foundation Project. http://qgis.osgeo.org

Rasmussen L. V., Fagan M. E., Ickowitz A., Wood S. L. R., Kennedy G., Powell B., et al., 2020. Forest pattern, not just amount, influences dietary quality in five African countries. Global Food Security, 25: 100331. https://doi.org/10.1016/i.gfs.2019.100331

Reidsma P., Tekelenburg T., Berg M., Van Den Alkemade R., 2006 Impacts of land-use change on biodiversity: An assessment of agricultural biodiversity in the European Union. Agriculture, Ecosystems \& Environment, 114 (1): 86-102. https://doi. org/10.1016/i.agee.2005.11.026

Rifai N. Khattabi A, Moukrim S, Arahou M, Rhazi L, 2018. Évaluation de la dynamique de l'occupation du sol dans la zone humide RAMSAR de Tahaddart (Nord-Ouest du Maroc). Revue d’Écologie (La Terre et la Vie), 73 (2) : 142-152. http://hdl.handle. net/2042/66101

Sangne C. Y., Sadaiou Y., Barima S., Bamba I., Doumé A. N., 2019. Dynamique forestière post-conflits armés de la Forêt classée du Haut-Sassandra (Côte d'Ivoire). Vertig0, 15 (3) : 1-18. https://doi. org/10.4000/vertigo.16784

Sikuzani Y. U., Boisson S., Kaleba S. C., Khonde C. N., Malaisse F., Halleux J.-M., et al., 2019. Dynamique de l'occupation du sol autour des sites miniers : analyse à long terme de la structure spatiale à Lubumbashi. Biotechnologie, Agronomie, Société et Environnement / Biotechnology, Agronomy, Society and Environment, 24 (1) : 14-27. https://doi.org/10.25518/1780-4507.18306
Tchatchou B., Sonwa D. J, Ifo S., Tiani A. M., 2015. Déforestation et dégradation des forêts dans le Bassin du Congo : État des lieux, causes actuelles et perspectives. CIFOR, 47 p. https:// archive.pfbc-cbfp.org/actualites/items/D\%C3\%A9forestationd\%C3\%A9gradation.html

Temgoua L. F., Allaissem B., Tchamba M., Saradoum G., Osée M. M., Caroline M., Solefack M., 2018. Spatio-Temporal Dynamic of Land Use and Land Cover in the Classified Forest of Djoli-Kera, South-Eastern, Chad. Journal of Forestry, 8: 283-296. https://doi. org/10.4236/ojf.2018.83019

Tidjani M. A., Akponikpe P. B. I., 2012. Évaluation des stratégies paysannes d'adaptation aux changements climatiques: cas de la production du maïs au Nord-Bénin. African Crop Science Journal, 20 (2): 425-441. https://www.ajol.info/index.php/acsi/article/ view/81784

UNFCC, 2014. Forests Action Statements and Action Plans. The New York Declaration on Forests. Climate summit 2014, United nations, 18 p. https://unfccc.int/sites/default/files/new-york-declarationon-forests_26-nov-2015.pdf

Wasseige C. de, Devers D., de Marcken P., Eba'a Atyi R., Mayaux P., 2009. Les forêts du Bassin du Congo. État des forêts 2008. Luxembourg, Office des publications de l>Union européenne, $426 \mathrm{p}$. https://doi.org/10.2788/32456

White F., 1983. The vegetation of Africa. Paris, France, UNESCO, Natural Resources Research, 20, 356 p.

Yeo K., Tiho S., Ouattara K., Konate S., Maurice L. M., 2013. Impact de la fragmentation et de la pression humaine sur la relique forestière de l'Université d'Abobo-Adjamé (Côte d'Ivoire). Journal of Applied Biosciences, $61:$ 4551-4565.

\begin{tabular}{|c|c|}
\hline Rôle du contributeur & Noms des auteurs \\
\hline Conceptualisation & $\begin{array}{l}\text { F. C. Ahononga, G. N. Gouwakinnou, } \\
\text { S. S. H. Biaou, S. Biaou }\end{array}$ \\
\hline Gestion des données & F. C. Ahononga \\
\hline Analyse formelle & F. C. Ahononga \\
\hline $\begin{array}{l}\text { Acquisition du } \\
\text { financement }\end{array}$ & F. C. Ahononga \\
\hline $\begin{array}{l}\text { Enquête et } \\
\text { investigation }\end{array}$ & F. C. Ahononga, S. Biaou \\
\hline Méthodologie & $\begin{array}{l}\text { F. C. Ahononga, G. N. Gouwakinnou, } \\
\text { S. S. H. Biaou }\end{array}$ \\
\hline Gestion de projet & F. C. Ahononga, G. N. Gouwakinnou \\
\hline Ressources & F. C. Ahononga \\
\hline Logiciels & $\begin{array}{l}\text { F. C. Ahononga, G. N. Gouwakinnou, } \\
\text { S. S. H. Biaou, S. Biaou }\end{array}$ \\
\hline Supervision & F. C. Ahononga, S. S. H. Biaou \\
\hline Validation & $\begin{array}{l}\text { F. C. Ahononga, G. N. Gouwakinnou, } \\
\text { S. S. H. Biaou, S. Biaou }\end{array}$ \\
\hline Visualisation & $\begin{array}{l}\text { F. C. Ahononga, G. N. Gouwakinnou, } \\
\text { S. S. H. Biaou, S. Biaou }\end{array}$ \\
\hline $\begin{array}{l}\text { Écriture - Préparation } \\
\text { de l'ébauche originale }\end{array}$ & $\begin{array}{l}\text { F. C. Ahononga, G. N. Gouwakinnou, } \\
\text { S. Biaou }\end{array}$ \\
\hline $\begin{array}{l}\text { Écriture - Révision } \\
\text { et édition }\end{array}$ & $\begin{array}{l}\text { F. C. Ahononga, G. N. Gouwakinnou, } \\
\text { S. S. H. Biaou, S. Biaou }\end{array}$ \\
\hline
\end{tabular}

Bois et Forêts des Tropiques - Revue scientifique du Cirad (C) Bois et Forêts des Tropiques (c) Cirad
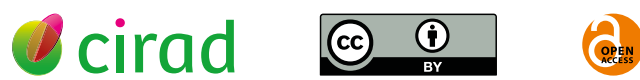

Cirad - Campus international de Baillarguet, 34398 Montpellier Cedex 5, France - Contact : bft@cirad.fr - ISSN : L-0006-579X 